\title{
A BEHAVIORAL ASSET PRICING MODEL WITH A TIME-VARYING SECOND MOMENT
}

\author{
CARL CHIARELLA*, XUE-ZHONG HE* AND DUO WANG ${ }^{\dagger}$ \\ *School of Finance and Economics \\ University of Technology, Sydney \\ PO Box 123 Broadway \\ NSW 2007, Australia \\ ${ }^{\dagger}$ LMAM \\ Department of Financial Mathematics \\ School of Mathematical Sciences \\ Peking University \\ Beijing 100871 \\ People's Republic of China
}

\begin{abstract}
We develop a simple behavioral asset pricing model with fundamentalists and chartists in order to study price behavior in financial markets when chartists estimate both conditional mean and variance by using a weighted averaging process. Through a stability, bifurcation, and normal form analysis, the market impact of the weighting process and time varying second moment are examined. It is found that the fundamental price becomes stable (unstable) when the activities from both types of traders are balanced (unbalanced). When the fundamental price becomes unstable, the weighting process leads to different price dynamics, depending on whether the chartists act as either trend followers or contrarians. It is also found that a time varying second moment of the chartists does not change the stability of the fundamental price, but it does influence the stability of the bifurcations. The bifurcation becomes stable (unstable) when the chartists are more (less) concerned about the market risk characterized by the time varying second moment. Different routes to complicated price dynamics are also observed. The analysis provides an analytical foundation for the statistical analysis of the corresponding stochastic version of this type of behavioral model.
\end{abstract}

JEL classifications: D83; D84; E21; E32, C60

Keywords: Fundamentalists, chartists, stability, bifurcation, normal form analysis, investors' under- and over-reactions, stylized facts.

Date: First Version: Jan. 2003; Second Version: Oct.6, 2004; Revised Version: June 3, 2005. Financial support for Duo Wang by NSFC.10271007 and RFDP.20010001042 and for Chiarella and He by ARC DP0450526 is acknowledged. This paper was prepared when Wang was visiting the Quantitative Finance Research Centre at the University of Technology, Sydney, whose hospitality is gratefully acknowledged. The authors would like to thank the referee for his/her helpful suggestions. The authors are also indebted to Dr. Hing Hung for having produced the bifurcation plots. 


\section{INTRODUCTION}

As a result of a growing dissatisfaction with models of asset price dynamics based on the representative agent paradigm, as expressed for example by Kirman (1992), and also the extreme informational assumptions of rational expectations, research into the dynamics of financial asset prices that result from the interaction of heterogeneous agents has developed strongly over the last two decades. ${ }^{1}$ In particular, Brock and Hommes $(1997 b, 1998)$ have introduced the concept of an adaptively rational equilibrium, where agents adapt their beliefs over time by choosing from different predictors or expectations functions, based upon their fitness functions measured by realized profits. The resulting dynamical system is capable of generating the entire zoo of complex behaviour from local stability to high order cycles and chaos as various key parameters of the model change. The Brock and Hommes framework has been extended further in Gaunersdorfer (2000) and Chiarella and He (2001, 2002, 2003b) to incorporate timevarying (homogeneous) variance and heterogeneous risk and learning under both the Walrasian auctioneer and market maker scenarios. It is found that the relative risk attitudes, different learning mechanisms and different market clearing scenarios affect asset price dynamics in a very complicated way. It has been shown (e.g. Hommes (2002)) that such simple nonlinear adaptive models are capable of explaining important stylized facts, including fat tails, volatility clustering and long memory, of real financial time series.

It is well recognised that heterogeneous expectations play a key role in the dynamical behaviour of asset prices. Most of the literature cited above focuses on heterogeneous expectations of the first moment (of either price or return), rather than the second moment. Empirically, it is believed that ${ }^{2}$ second moments (i.e. conditional variances) are much easier to estimate than first moments (i.e. conditional mean) and hence there should be more disagreement about the mean than about the variance among traders. Mathematically, the second moments are associated with higher order terms and they do not in general change the nature of local stability and bifurcations. However, when traders are heterogeneous, they may be heterogenous not only in their beliefs about the first moment but also about the second moment. This heterogeneity may come from their different information sets or the different trading strategies they are using. The aim of this paper is to study the dynamical behaviour of prices when traders are heterogeneous with respect to both moments.

Given the variety of technical trading rules and differing risk aversion of various investors, this paper introduces a risk adjustment into the demand function for the chartists by assuming that they use a weighted average process of past prices to estimate and update both conditional mean and variance. Therefore their demand function is a nonlinear function of the conditional mean and variance, instead of a linear function of the conditional mean only. It is found that the mechanism of a variance adjusted demand function of the chartists is a natural way to prevent the price from

\footnotetext{
${ }^{1}$ See, for example Arthur et al. (1997), Brock and Hommes (1997a, 1997b), Brock and LeBaron (1996), Bullard and Duffy (1999), Chen and Yeh (1997, 2002), Chiarella (1992), Chiarella et al. (2002), Chiarella and He (2001, 2002, 2003b), Dacorogna et al. (1995), Day and Huang (1990), Farmer and Joshi (2002), Gaunersdorfer (2000), Gaunersdorfer et al. (2003), Hommes (2001, 2002), LeBaron et al. (1999), Lux (1995, 1997, 1998) and Lux and Marchesi (1999).

${ }^{2}$ See, for example, Nelson (1992) and Bollerslev et al. (1994) for some justification of this assertion.
} 
getting stuck in a speculative bubble ${ }^{3}$. Similar to Brock and Hommes (1997b, 1998), an adaptive model based on a fitness function is obtained. We then examine how the price dynamics of the risky asset are affected by the reactions of investors, the switching intensity of the fitness function, and the weighting process and risk adjustment of the chartists.

This paper is closely related to Gaunersdorfer et al. (2003) who consider a simple asset pricing model of fundamentalists and chartists. In their model, the fundamentalists believe that tomorrow's price will move in the direction of the fundamental price, while chartists derive their beliefs from a simple technical trading rule using only the latest observed price (rather than a weighted average of the latest history of prices as in the current paper) and extrapolation of the latest observed price change. They assume a homogeneous time-varying conditional variance for both types of traders (as opposed to a constant variance for the fundamentalists and the adjusted time-varying variance which is estimated from the weighted average process for the chartists in this paper) and impose a penalty function in the fitness function of the chartists (to ensure that speculative bubbles cannot last forever). They go on to show that volatility clustering can be characterized by the coexistence of a stable steady state and a stable limit cycle, which arises as a consequence of a so-called Chenciner bifurcation.

To study the impact of the time-varying second moment, we use a normal form analysis associated with the standard stability and bifurcation analysis. Our analysis shows that, when the fundamental price becomes unstable, the time varying second moment and the weighting process lead to different price dynamics. This is due to the fact that the loss of local stability is accompanied by either flip or Hopf bifurcations, depending on whether the chartists act as either trend followers or contrarians. The time-varying second moment has no influence on the stability of the fundamental price. However, it does affect the stability of the bifurcations, which are stable (unstable) when the chartists are more (less) concerned about the time-varying second moment. Different routes to complicated price dynamics are also observed. The analysis provides an analytical foundation for the statistical analysis of the corresponding stochastic version of this behavioural model studied in Chiarella et al. (2005).

The plan of the paper is as follows. Section 2 develops a simple fundamentalist and chartist asset pricing model in a Walrasian market clearing scenario. The dynamics of the deterministic system, including stability and bifurcation analysis, when chartists are either trend followers or contrarians are examined in Sections 3 and 4, respectively. Section 5 concludes. All proofs are included in the Appendix.

\section{THE MODEL}

2.1. Portfolio Optimization and Walrasian Equilibrium Price. Following the framework of Brock and Hommes (1998), consider an asset pricing model with one risky asset and one risk free asset. It is assumed that the risk free asset is perfectly elastically supplied at the risk-free (annualised) rate $r$. Let $p_{t}$ be the price (ex dividend) per share

\footnotetext{
${ }^{3}$ In Gaunersdorfer et al. (2003), this is ensured by artificially adding a penalty function in the fitness function of the chartists, which is unnecessary in the model studied in this paper. A similar stabilizing force is added to the fitness function for the fundamentalists in Gaunersdorfer (2000) where a homogeneous time-varying second moment is updated through an exponential moving average process.
} 
of the risky asset at time $t$ and $\left\{y_{t}\right\}$ be the stochastic dividend process of the risky asset. Then the wealth of investor $h$ at $t+1$ is given by

$$
W_{h, t+1}=R W_{h, t}+\left(p_{t+1}+y_{t+1}-R p_{t}\right) z_{h, t}=R W_{h, t}+R_{t+1} z_{h, t},
$$

where

$$
R_{t+1}=p_{t+1}+y_{t+1}-R p_{t}
$$

is the excess capital gain/loss, $R=1+r / K, K$ is the trading frequency per annum ${ }^{4}$, $W_{h, t}$ is the wealth at time $t$ and $z_{h, t}$ is the number of shares of the risky asset purchased at $t$. Denote by $F_{t}=\left\{p_{t-1}, \cdots ; y_{t-1}, \cdots\right\}$ the information set available at time $t$ to all investors. Let $E_{h, t}, V_{h, t}$ be the beliefs of investor type $h$ about the conditional expectation and variance, based on $F_{t}$. Then it follows from (2.1) and (2.2) that

$$
E_{h, t}\left(W_{t+1}\right)=R W_{t}+E_{h, t}\left(R_{t+1}\right) z_{h, t}, \quad V_{h, t}\left(W_{t+1}\right)=z_{h, t}^{2} V_{h, t}\left(R_{t+1}\right) .
$$

Assume each investor has a CARA (constant absolute risk aversion) utility function $u(W)=-e^{-a_{h} W}$ but with different risk aversion coefficient $a_{h}$, and maximises his/her expected utility of wealth, leading to the optimal demand

$$
z_{h, t}=\frac{E_{h, t}\left(R_{t+1}\right)}{a_{h} V_{h, t}\left(R_{t+1}\right)} .
$$

As in Brock and Hommes (1998), a Walrasian scenario is used to derive the demand equation, i.e. each trader is viewed as a price taker (see Brock and Hommes (1997a) and Grossman (1989) for detailed discussion). The market is viewed as finding the price $p_{t}$ that equates the sum of these demand schedules to the supply. Let $z_{s, t}$ denote the supply of (risky) shares. Given bounded rationality, we classify all investors into $H$ different types in terms of their conditional expectations on both mean and variance. Denote by $n_{h, t}$ the fraction of investors of type $h$ at $t$ (so that $\sum_{h} n_{h, t}=1$ ). Then the equilibrium of demand and supply implies

$$
\sum_{h} n_{h, t} \frac{E_{h, t}\left(R_{t+1}\right)}{a_{h} V_{h, t}\left(R_{t+1}\right)}=z_{s t} .
$$

Now assume zero supply of outside shares, i.e. $z_{s t}=0$, then (2.5) leads to

$$
\sum_{h} n_{h, t} \frac{E_{h t}\left(R_{t+1}\right)}{a_{h} V_{h t}\left(R_{t+1}\right)}=0 .
$$

Under the conditions that $E_{t}\left(y_{t+1}\right)=\bar{y}$ and $\lim _{t \rightarrow \infty} E p_{t} / R^{t}=0$, it can be shown that the fundamental price is constant and given by $p^{*}=\bar{y} /(R-1)$, which corresponds to the benchmark notion of the rational expectation; see Brock and Hommes (1998) for related discussion.

2.2. Heterogeneous Beliefs. In the following discussion, we adopt the much analyzed fundamentalist/chartist model by assuming that all investors can be grouped as either fundamentalists (type 1) or chartists (type 2).

${ }^{4}$ Typically, annually, quarterly, monthly, weekly and daily trading periods correspond to $K=$ $1,4,12,52$ and 250 , respectively. 
2.2.1. Fundamentalists. The fundamentalists are assumed to believe that the expected market price $p_{t+1}$ is mean reverting to their perceived fundamental value $p^{*}$ and that the conditional variance of the market price is constant. That is,

$$
\left\{\begin{array}{l}
E_{1, t}\left(p_{t+1}\right)=p^{*}+v\left(p_{t-1}-p^{*}\right), \quad 0 \leq v \leq 1 \\
V_{1, t}\left(p_{t+1}\right)=\sigma_{1}^{2}
\end{array}\right.
$$

where $p^{*}$ is the fundamental price of the risky asset estimated by the fundamentalists at some cost, $v$ is the speed of mean reversion estimated by the fundamentalists, and $\sigma_{1}>0$ is a constant. In particular, $E_{1, t}\left(p_{t+1}\right)=p^{*}$ for $v=0$ and $E_{1, t}\left(p_{t+1}\right)=p_{t-1}$ for $v=1$. The conditional expectation of the fundamentalists (2.7) can also be written as

$$
E_{1, t}\left(p_{t+1}\right)=(1-v) p^{*}+v p_{t-1}, \quad 0 \leq v \leq 1,
$$

which is a weighted average of the fundamental price and latest price. Hence small (large) values of $v$ indicate that the fundamentalists give more (less) weight to the fundamental price and less (more) weight to the latest price, believing that price moves quickly (slowly) towards its fundamental value $p^{*}$. For convenience of discussion, we say the fundamentalists over(under)-react (to the market price) when more (less) weight $v$ is given to the market price.

2.2.2. Chartists. Unlike the fundamentalists who are able to determine (at some cost) the fundamental value, chartists base their trading strategy on signals generated from the costless information contained in recent prices. The signal may be generated by comparing the latest price $p_{t-1}$ with some reference price trend $\tilde{p}_{t-1}$ which chartists assume is randomly drawn from some distribution conditional on past realized prices. For simplicity, we assume that $\tilde{p}_{t-1}$ is conditionally distributed on prices $p_{t-2}$ and $p_{t-3}$ with weighting probabilities $w$ and $1-w$, respectively. Then the conditional mean and variance of the trend can be estimated by, respectively,

$$
\left\{\begin{aligned}
\bar{p}_{t-2} & :=E_{2, t}\left(\tilde{p}_{t-1} \mid p_{t-2}, p_{t-3}\right)=w p_{t-2}+(1-w) p_{t-3}, \quad 0 \leq w \leq 1, \\
\bar{\sigma}_{t-2}^{2} & :=V_{2, t}\left(\tilde{p}_{t-1} \mid p_{t-2}, p_{t-3}\right)=w\left[p_{t-2}-\bar{p}_{t-2}\right]^{2}+(1-w)\left[p_{t-3}-\bar{p}_{t-2}\right]^{2} .
\end{aligned}\right.
$$

Based on the trading signals $p_{t-1}-\tilde{p}_{t-1}$ and the conditional mean and variance estimates (2.8), we make the following assumptions about the chartists:

$$
\left\{\begin{array}{l}
E_{2, t}\left(p_{t+1}\right)=p_{t-1}+g\left(p_{t-1}-\bar{p}_{t-2}\right), \quad g \in \mathbb{R}, \\
V_{2, t}\left(p_{t+1}\right)=\sigma_{1}^{2}\left[1+b \bar{\sigma}_{t-2}^{2}\right], \quad b \geq 0,
\end{array}\right.
$$

where $g \in \mathbb{R}$ is the estimated extrapolation rate of the chartists. That is, the chartists' beliefs are based on the latest price and their extrapolation of the trading signals generated from the trend. In particular, chartists are called trend followers when $g>0$ and are contrarians when $g<0$. For $w=1, E_{2, t}\left(p_{t+1}\right)=p_{t-1}+g\left(p_{t-1}-p_{t-2}\right)$ which is the case discussed in Gaunersdorfer et al. (2003) and, for $w=0, E_{2, t}\left(p_{t+1}\right)=$ $p_{t-1}+g\left(p_{t-1}-p_{t-3}\right)$. Similarly, for convenience of discussion, we say the chartists over(under)-react when they extrapolate strongly (weakly), that is when $|g|$ is large (small). With regard to the chartists' estimate of the variance, they use the historical variance to scale up the fundamental variance through the parameter $b$. High $b$ reflects a greater sensitivity to risk. 
2.2.3. Optimal Demand for the Fundamentalists and Chartists. For the dividend process, we assume that this is common knowledge, so that for all agents

$$
E\left(y_{t}\right)=\bar{y}, \quad V\left(y_{t}\right)=\sigma_{y}^{2},
$$

where we set $\bar{y}=r p^{*} / K$. Based on the above assumptions, one obtains that for the fundamentalists

$$
\left\{\begin{array}{l}
E_{1, t}\left(R_{t+1}\right)=p^{*}+v\left(p_{t-1}-p^{*}\right)+\bar{y}-R p_{t}, \quad 0 \leq v \leq 1 \\
V_{1, t}\left(R_{t+1}\right)=\sigma_{1}^{2}+\sigma_{y}^{2}
\end{array}\right.
$$

and for the chartists

$$
\left\{\begin{array}{l}
E_{2, t}\left(R_{t+1}\right)=p_{t-1}+g\left(p_{t-1}-\bar{p}_{t-2}\right)+\bar{y}-R p_{t}, \quad g \in \mathbb{R} \\
V_{2, t}\left(R_{t+1}\right)=\sigma_{y}^{2}+\sigma_{1}^{2}\left[1+b \bar{\sigma}_{t-2}^{2}\right], \quad b \geq 0
\end{array}\right.
$$

Therefore, the optimal demands for the fundamentalists and chartists are given, respectively, by

$$
\left\{\begin{array}{l}
z_{1, t}=\left[p^{*}+v\left(p_{t-1}-p^{*}\right)+\bar{y}-R p_{t}\right] / A_{1} \\
z_{2, t}=\left[p_{t-1}+g\left(p_{t-1}-\bar{p}_{t-2}\right)+\bar{y}-R p_{t}\right] / A_{2, t}
\end{array}\right.
$$

where

$$
A_{1}=a_{1}\left(\sigma_{1}^{2}+\sigma_{y}^{2}\right), \quad A_{2, t}=a_{2}\left[\sigma_{y}^{2}+\sigma_{1}^{2}\left(1+b \bar{\sigma}_{t-2}^{2}\right)\right]
$$

and $\bar{p}_{t}$ and $\bar{\sigma}_{t}^{2}$ are defined by (2.8).

2.3. Performance Measure and Agent Adaptation. Let $U_{1, t}$ and $U_{2, t}$ be the realized profit of the fundamentalists and chartists, respectively, defined by

$$
U_{i, t}=R_{t} z_{i, t-1}-C_{i}, \quad i=1,2
$$

where $C_{i} \geq 0$ measures the total cost. Let the updated fractions be formed on the basis of discrete choice probability (see Manski and McFadden (1981), Anderson, de Palma and Thisse (1993), Brock and Hommes (1997b, 1998), so that

$$
n_{i, t}=\exp \left[\beta U_{i, t-1}\right] / Z_{t-1}, \quad(i=1,2), \quad Z_{t-1}=\sum_{i=1}^{2} \exp \left[\beta U_{i, t-1}\right],
$$

where $\beta(>0)$ is the intensity of choice measuring how fast agents switch among different prediction strategies. ${ }^{5}$ Let $m_{t}=n_{1, t}-n_{2, t}$. Then $n_{1, t}=\left(1+m_{t}\right) / 2, n_{2, t}=$ $\left(1-m_{t}\right) / 2$ and

$$
\begin{aligned}
m_{t} & =\tanh \left[\frac{\beta}{2}\left(U_{1, t-1}-U_{2, t-1}\right)-\frac{\beta}{2}\left(C_{1}-C_{2}\right)\right] \\
& =\tanh \left[\frac{\beta}{2} R_{t-1}\left(z_{1, t-2}-z_{2, t-2}\right)-\frac{\beta}{2} C\right],
\end{aligned}
$$

where $C=C_{1}-C_{2}$. Because the cost of information to work out the fundamental values, the constant $C \geq 0$ in general.

\footnotetext{
${ }^{5}$ To prevent the price from getting stuck in a speculative bubble, Gaunersdorfer (2000) introduces a stabilizing force into the fitness function $U_{i, t}$, while Gaunersdorfer et al. (2003) add a penalty function in the changing population fraction. In our model, this is achieved naturally by incorporating the conditional variance into the demand function of the chartists.
} 
2.4. The Complete Model. To sum up, assuming zero net supply the Walrasian equilibrium price $p_{t}$ satisfies

$$
\left(1+m_{t}\right) z_{1, t}+\left(1-m_{t}\right) z_{2, t}=0 .
$$

Substituting (2.13) and (2.16) into (2.17), one obtains that

$$
p_{t}=\frac{A_{2, t}\left[p^{*}+v\left(p_{t-1}-p^{*}\right)+\bar{y}\right] e^{\beta U_{t-1}}+A_{1}\left[p_{t-1}+g\left(p_{t-1}-\bar{p}_{t-2}\right)+\bar{y}\right]}{R\left[A_{2, t} e^{\beta U_{t-1}}+A_{1}\right]},
$$

where

$$
\begin{aligned}
U_{t}= & R_{t}\left(z_{1, t-1}-z_{2, t-1}\right)-C \\
= & \left(p_{t}+y_{t}-R p_{t-1}\right)\left(\frac{1}{A_{1}}\left[p^{*}+v\left(p_{t-2}-p^{*}\right)+\bar{y}-R p_{t-1}\right]\right. \\
& \left.\quad-\frac{1}{A_{2, t-1}}\left[p_{t-2}+g\left(p_{t-2}-\bar{p}_{t-3}\right)+\bar{y}-R p_{t-1}\right]\right)-C,
\end{aligned}
$$

and $\bar{p}_{t}, A_{1}$ and $A_{2, t}$ are defined by (2.8) and (2.14). Equation (2.18) determines the Walrasian market cleaning price $p_{t}$, which is summarized in the following Proposition 2.1.

Proposition 2.1. Denote $x_{1, t}=x_{t}=p_{t}-p^{*}, x_{2, t}=x_{t-1}, x_{3, t}=x_{t-2}, x_{4, t}=x_{t-3}$ and $x_{5, t}=x_{t-4}$. Then the market clearing price $p_{t}=x_{t}+p^{*}$ is governed by the following dynamical system in terms of $X=\left(x_{1}, x_{2}, x_{3}, x_{4}, x_{5}\right)^{T}$ :

$$
X_{t}=G\left(X_{t-1}\right)
$$

where

$$
\begin{aligned}
G(X)= & \left(F(X), x_{1}, x_{2}, x_{3}, x_{4}\right)^{T} \\
F(X)= & f_{1}(X) / f_{2}(X), \\
f_{1}(X)= & v A_{21} x_{1} e^{\beta U(X)}+A_{1}\left(x_{1}+g\left(x_{1}-w x_{2}-(1-w) x_{3}\right)\right), \\
f_{2}(X)= & R\left[A_{21} e^{\beta U(X)}+A_{1}\right] \\
U(X)= & \left(x_{1}-R x_{2}\right)\left(\frac{v x_{3}-R x_{2}}{A_{1}}\right. \\
& \left.-\frac{x_{3}+g\left(x_{3}-w x_{4}-(1-w) x_{5}\right)-R x_{2}}{A_{22}}\right)-C
\end{aligned}
$$

and

$$
\begin{array}{ll}
A_{1}=a_{1}\left(\sigma_{y}^{2}+\sigma_{1}^{2}\right), & A_{21}=a_{2}\left[\sigma_{y}^{2}+\sigma_{1}^{2}\left(1+b \bar{\sigma}_{21}^{2}\right)\right], \quad A_{22}=a_{2}\left[\sigma_{y}^{2}+\sigma_{1}^{2}\left(1+\bar{\sigma}_{22}^{2}\right)\right], \\
\bar{x}_{21}=w x_{2}+(1-w) x_{3}, & \bar{\sigma}_{21}^{2}=w\left[x_{2}-\bar{x}_{21}\right]^{2}+(1-w)\left[x_{3}-\bar{x}_{21}\right]^{2}, \\
\bar{x}_{22}=w x_{4}+(1-w) x_{5}, & \bar{\sigma}_{22}^{2}=w\left[x_{4}-\bar{x}_{22}\right]^{2}+(1-w)\left[x_{5}-\bar{x}_{22}\right]^{2} .
\end{array}
$$

In addition, the population fraction difference $m_{t}=n_{1, t}-n_{2, t}$ evolves according to

$$
m_{t}:=M\left(X_{t-1}\right)=\tanh [\beta U(X) / 2] .
$$

In the following discussion, we examine the dynamics of the nonlinear deterministic system (2.19) by considering the chartists as either trend followers or contrarians. We first undertake a theoretical study of the existence of the steady state, its stability, 
bifurcation, and normal form properties, followed by numerical simulations of the nonlinear system (2.19) to obtain some insight into its global properties.

\section{Dynamics of Fundamentalists ANd TREND Followers}

In this section, we consider the situation in which the chartists are trend followers, that is $g>0$ in model (2.19). We first have the following result on the existence of steady state that corresponds to the fundamental price $p^{*}$.

Proposition 3.1. For system (2.19), $X^{*}=0$ is the unique steady state and it corresponds to the fundamental price $p_{t}=p^{*}$.

Proof. See Appendix A.1.

Results on the local stability of the unique steady state and its bifurcation when the chartists are trend followers are given in the following Proposition.

Proposition 3.2. Assume $g>0$. Then the fundamental price $p^{*}$ is locally asymptotically stable (LAS) if

- either

$$
v<v_{3}(g):=\frac{c_{1}}{g}-c_{2}-c_{3} g \quad \text { for } \quad w \in[0,1),
$$

- or $g<g_{1}:=\left(a+x_{o}\right) R / a$ for $w=1$,

where

$$
\begin{aligned}
& c_{1}=\frac{\left(x_{0}+a\right)^{2} R^{2}}{a x_{0}(1-w)}, \quad c_{2}=\frac{x_{0} w R+a w R+a(1-w)}{x_{0}(1-w)}, \\
& c_{3}=\frac{a(2-w)}{x_{0}}, \quad x_{0}=e^{-\beta C}, \quad a=\frac{a_{1}}{a_{2}} .
\end{aligned}
$$

If $v=v_{3}(g)$ for $w \in[0,1)$ or $g=g_{1}$ for $w=1$, then there exists a pair of complex eigenvalues $\lambda=e^{ \pm i \theta}$ for the linearized system of (2.19) at the steady state. If, in addition, $d \neq 0$, where

$$
d:=\frac{1}{2} \frac{1}{e^{-3 i \theta}-e^{-i \theta}+\gamma_{3}\left(1-e^{-2 i \theta}\right)} \sum_{j, k, l=1}^{5} \frac{\partial^{3} F(0)}{\partial x_{j} \partial x_{k} \partial x_{l}} e^{(j+k-l-5) i \theta}
$$

and

$$
\gamma_{3}=-\frac{g a(1-w)}{R\left(x_{o}+a\right)}
$$

then, in the absence of strong resonances (i.e., $e^{i k \theta} \neq 1$ for $k=1,2,3,4$ ), a Hopf (Neimark-Sacker) bifurcation emanates out of the steady state $X^{*}=0$. Furthermore the bifurcation is supercritical when $d<0$ and subcritical when $d>0$.

Proof. See Appendix A.2.

The local stability region of the steady state and the corresponding Hopf bifurcation boundary are plotted in the $(g, v)$ parameter plane in Fig. 3.1. It can be verified that, along the Hopf bifurcation curve, $g \rightarrow g_{1}$ as $w \rightarrow 1^{6}$. In this limiting case, the Hopf

${ }^{6}$ It follows from $v=v_{3}(g)$ that $g=v_{3}^{-1}(v)$. Then it can be verified that $g \rightarrow g_{1}$ as $w \rightarrow 1$ 


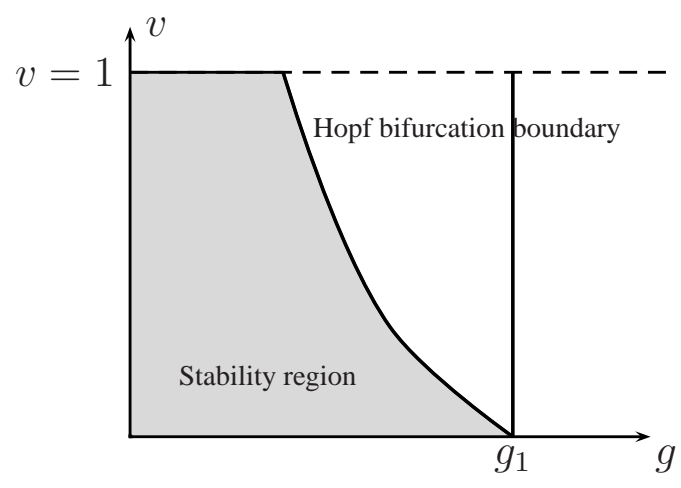

FIGURE 3.1. Local stability region of the steady state and the Hopf bifurcation boundary for $g>0$.

bifurcation boundary is independent of the speed of price adjustment of the fundamentalists. In general, for $w \in[0,1)$, Proposition 3.2 indicates that the condition for the Hopf bifurcation depends on the speed of price adjustment of the fundamentalists, the extrapolation rate of the trend followers, and the weighting process used by the trend followers to form the moving average ${ }^{7}$. Economically, reaction from the fundamentalists represents a stabilizing force while extrapolation from the chartists represents a destabilizing force. However, over-reaction from the fundamentalists can destabilize the fundamental price and under-reaction (or extrapolation) from the trend followers can smooth and stabilize the market price. This double edge effect of the heterogeneous beliefs leads to many interesting and complicated market price dynamics. More precisely, we have the following observations:

- Along the bifurcation boundary, $v_{3}(g)$ decreases as $g$ increases and this is illustrated by the bifurcation value $g^{*}$ in Table 3.1 for fixed $w=0.5$ and different values of $v$. This implies that the fundamental steady state price is locally stable as long as reactions of both types of investors are balanced. Our results show that the market price converges to the fundamental price when either the price trend is extrapolated weakly by the chartists or the reaction of the fundamentalists to the price dispersion (from the fundamental price) is weak. Otherwise, over-reactions from both types of traders lead the market price to diverge from the fundamental price. Intuitively, this instability of the fundamental price, together with the interaction between stabilizing and destabilizing forces, leads the market price to fluctuate around the fundamental price. The same result is obtained in Gaunersdorfer et al. (2003) when $w=1$, which is a special case of our result.

- Based on the fact that $g=v_{3}^{-1}(v) \rightarrow g_{1}$ as $w \rightarrow 1$, we can see that the stability region of the fundamental steady state in $(g, v)$ parameter space is enlarged as $w$ increases, in particular, this becomes even more significant when $v$ is close to 1 , as verified by the bifurcation values $g^{*}$ in Table 3.2 for fixed

${ }^{7}$ See Appendix A.2 for detailed discussion. 
$v=0.5$ and different values of $w$. Economically, the market price converges to the fundamental price when more weight is given to the most recent price in forming price trend.

- Along the Hopf bifurcation boundary, the type of bifurcation depends on values of $\theta$ in the pair of complex eigenvalues $\lambda_{2,3}=e^{ \pm \theta i}$ of the linearized system at the steady state, which in turn depends on values of $\rho=2 \cos (\theta)$ satisfying $^{8}$

$$
\rho=\frac{R\left(x_{0}+a\right)-g a w}{g a(1-w)} .
$$

Tables 3.1 and 3.2 illustrate the Hopf bifurcation values $g^{*}$ and the corresponding $\rho$ for various $v$. Similar to the findings in Chiarella and He (2003a), depending on values of $\rho$ (and hence of $\theta$ ), periodic (when $\theta /(2 \pi)$ is a rational number) and quasi-periodic (when $\theta /(2 \pi)$ is an irrational number) cycles can be generated through the Hopf bifurcation. The stability of the bifurcated cycles depends on the invariant expression $d$ of the normal form analysis in Proposition 3.2. More details on normal form analysis in higher dimension can be found in Kuznetsov (2004).

Guided by the stability, bifurcation and normal form results in Proposition 3.2, we now turn to a numerical study of the dynamics of the nonlinear system (2.19). In the following, we choose the fundamental price $p^{*}=\$ 100$, annual risk-free rate $r=5 \%$, annual volatility of the fundamental price $\sigma=20 \%$. For the trading frequency, we choose $K=250$, which corresponds to a daily trading period. As a consequence ${ }^{9}$, the total risk-free return per trading day $R=1+r / K=1.0002$, daily price volatility $\sigma_{1}^{2}=\left(p^{*} \sigma\right)^{2} / K=8 / 5$ and daily dividend volatility $\sigma_{y}^{2}=r^{2} \sigma_{1}^{2}=1 / 250$. We also choose the risk aversion coefficients for both types of investor as $a_{1}=a_{2}=0.8$ and the cost difference $C=0$. We examine how the dynamics of the nonlinear system is affected by the speed of price adjustment of the fundamentalists (the parameter $v$ ), the extrapolation rate of the trend followers (the parameter $g$ ), the coefficient of the variance adjusted demand (the parameter $b$ ), the weighting parameter used by the trend followers (the parameter $w$ ), and the switching intensity (the parameter $\beta$ ).

\begin{tabular}{|c||c|c|c|c|c|c|c|c|c|c|c|}
\hline$v$ & 0 & 0.1 & 0.2 & 0.3 & 0.4 & 0.5 & 0.6 & 0.7 & 0.8 & 0.9 & 1 \\
\hline$g^{*}$ & 1.5169 & 1.4971 & 1.4775 & 1.4583 & 1.4395 & 1.4210 & 1.4029 & 1.3851 & 1.3676 & 1.3505 & 1.3337 \\
\hline$\rho$ & 1.63789 & 1.6725 & 1.7078 & 1.7434 & 1.7792 & 1.8154 & 1.8518 & 1.8884 & 1.9253 & 1.9625 & 2.0000 \\
\hline
\end{tabular}

TABLE 3.1. Hopf bifurcation values $g^{*}$ and the corresponding values of $\rho$ for various $v$ and $w=0.5, \beta=2, C=0$.

For fixed $w=0.5$, Table 3.1 lists the bifurcation values $g^{*}$ for various values of $v$. It clearly indicates that the fundamental price is always locally stable for any speed of price adjustment $v \in[0,1]$ when the extrapolation rate of the trend followers is below the critical value $g_{1}^{*}(=1.3337)$. When the extrapolation rate exceeds the critical value $g_{1}^{*}$, the stability region for the extrapolation rate of the trend followers is enlarged as

\footnotetext{
${ }^{8}$ See Appendix A.2 for the details.

${ }^{9} \mathrm{We}$ approximate the annual dividend volatility $\sigma_{Y}$ by $r\left(p^{*} \sigma\right)$ and hence the daily dividend volatility $\sigma_{y}$ by $r\left(p^{*} \sigma\right) / \sqrt{K}$.
} 
the fundamentalists under-react, as suggested by Proposition 3.1. Consequently, overreactions from both the trend followers and fundamentalists can make the fundamental price become unstable through a Hopf bifurcation.

\begin{tabular}{|c||c|c|c|c|c|c|c|c|c|c|c|}
\hline$w$ & 0 & 0.1 & 0.2 & 0.3 & 0.4 & 0.5 & 0.6 & 0.7 & 0.8 & 0.9 & 1 \\
\hline$g^{*}$ & 1.0883 & 1.1422 & 1.2016 & 1.2671 & 1.3400 & 1.4208 & 1.5118 & 1.6137 & 1.7284 & 1.8572 & 2.0004 \\
\hline$\rho$ & 1.8380 & 1.8348 & 1.8310 & 1.8270 & 1.8219 & 1.8156 & 1.8079 & 1.7986 & 1.7867 & 1.7711 & 1.7499 \\
\hline
\end{tabular}

TABLE 3.2. Hopf bifurcation values $g^{*}$ and the corresponding values of $\rho$ for various $w$ and $v=0.5, \beta=2, C=0$.

Similarly, for fixed $v=0.5$, Table 3.2 lists some Hopf bifurcation values $g^{*}$ for various values of $w$, indicating that an increase of the weighting parameter $w$ enlarges the stability region for the extrapolation rate of the trend followers. This implies that a simple ad hoc trend following strategy based on the difference of the latest two prices $\left[p_{t-1}-p_{t-2}\right]$ is better than a more sophisticated strategy based on the difference $p_{t-1}-\left[w p_{t-2}+(1-w) p_{t-3}\right]$ for the chartists to forecast the fundamental price.
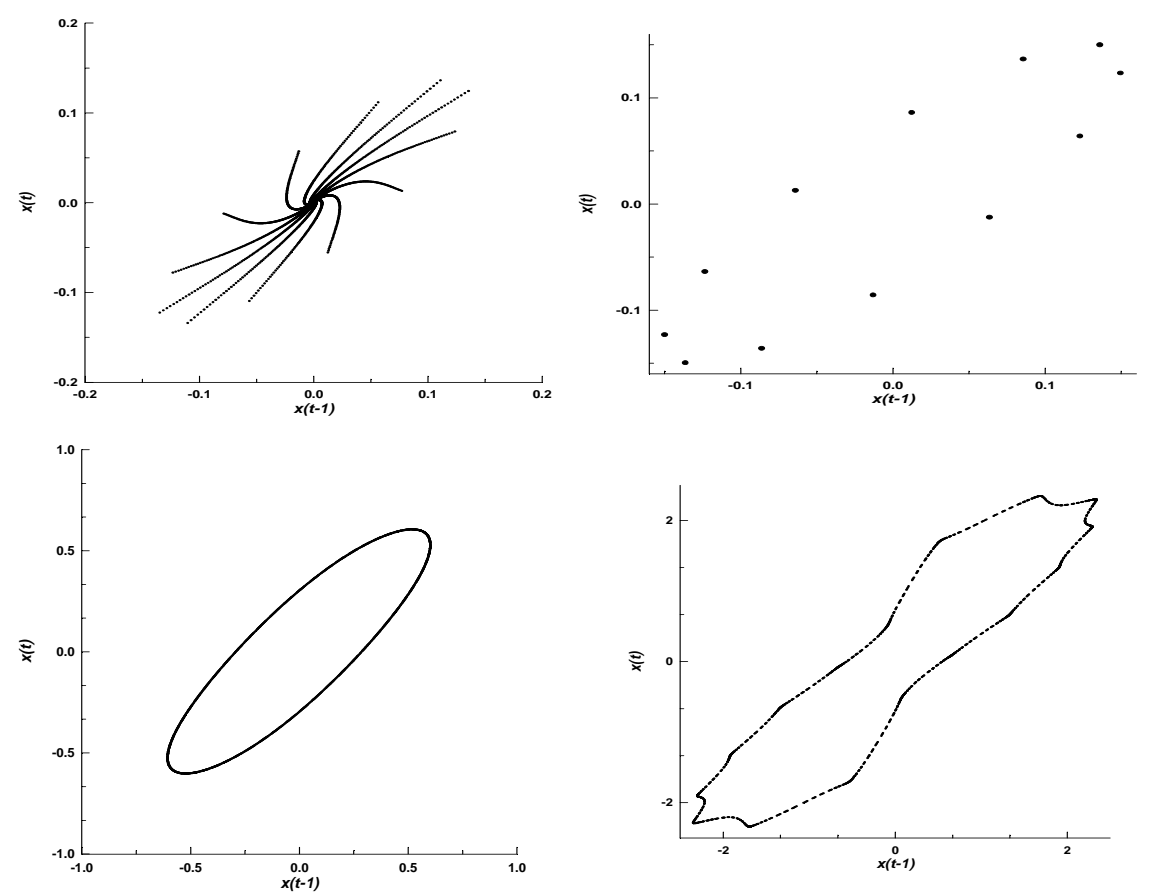

FIGURE 3.2. Phase plots of $\left(x_{t}, x_{t-1}\right)$ for $w=0.5, b=2, \beta=1, v=$ 0.2682 and four values of $g=1.46$ (upper left), $g^{*}$ (upper right), 1.48 (lower left), and 1.52 (lower right).

Proposition 3.2 states that the only bifurcation occurring at an unstable steady state is the Hopf bifurcation. The nature of the (quasi)-periodicity of the Hopf bifurcation is determined by values of $\theta$, which are in turn determined by values of $\rho$, displayed in Table 3.1. For example, let $\theta /(2 \pi)=1 / 12$, then $\rho=1.73205$ and $g^{*}=1.464395$, which corresponds to $v=0.2682$ with fixed $w=0.5$. To see the price dynamics near 
the Hopf bifurcation boundary, we choose $\beta=1, b=2, w=0.5, v=0.2682$ and four different values of $g=1.46, g^{*}, 1.48,1.52$. Fig. 3.2 gives the limiting phase plots in the $\left(x_{t-1}, x_{t}\right)$ plane for these four different values. For $g=1.46<g^{*}$, the upper left panel shows that the trajectories converge periodically to the steady state. For $g=g^{*}$, the upper right panel shows that the trajectories converge to a period-12 cycle, which verifies the above calculation. For $g=1.48>g^{*}$, the lower left panel shows that the trajectories converge to a quasi-periodic orbit, which is characterized by a closed orbit in the phase plane. For $g=1.52\left(>g^{*}\right)$, the lower right panel shows that the trajectories converge to a strange attractor.

Coexistence of a stable equilibrium and a stable closed curve has been detected in Gaunersdorfer et al. (2003) and they have found that such coexistence can be a potential mechanism in generating volatility clustering for the corresponding stochastic model. For the set of parameters choose above, in the local stability region, we are not able to detect the coexistence of another attractor. However, near the bifurcation boundary, coexistence of closed cycles with differential initial values for $g=1.4664$ is found, as illustrated in Fig. 3.3 (a) ${ }^{10}$. This coexistence of different cycles is different from that in Gaunersdorfer et al. (2003). Whether such coexistence can be a potential mechanism in generating volatility clustering would be an interesting issue for the future work. The corresponding bifurcation plot for the parameter $g$ is given in Fig. 3.3(b). This illustrates one of many routes to complicated price dynamics through the Hopf bifurcation. Economically, high price volatility is expected when the price trend is extrapolated strongly by the chartists and this verifies the common belief that the chartists represent an unstable force in financial markets.

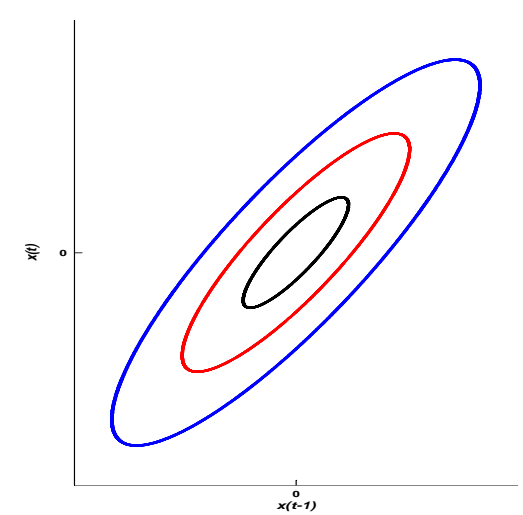

(a)

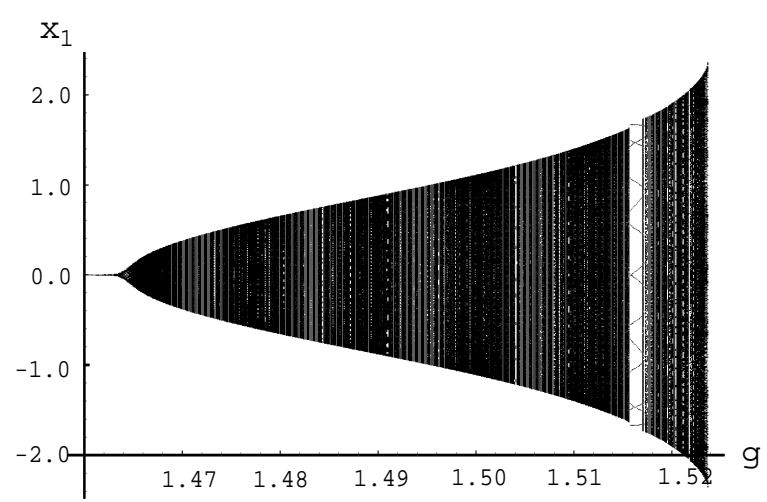

(b)

FIGURE 3.3. (a) Coexistence of closed cycles with differential initial values and parameter $g=1.4664$; (b) Bifurcation plot for the parameter $g$. The other parameters are $w=0.5, b=2, \beta=1, v=0.2682, C=0$.

The effect of the switching intensity $\beta$ on the price dynamics displays the wellknown rational routes to randomness observed in Brock and Hommes (1998). That is, when agents adapt their belief quickly to a better performing strategy, this rational

\footnotetext{
${ }^{10}$ The authors would like to thank the referee to bring this issue to our attention.
} 
adaption can make the market price become more volatile, leading to more complicated, and even explosive behaviour.

We now examine the effect of the variance adjustment coefficient $b$ of the trend followers (see equation (2.9)). Note that the local stability conditions are independent of the variance coefficient $b$. However, the normal form analysis indicates that the existence and stability of the bifurcated cycle turn out to depend on $b$. This observation leads to a very interesting result — changes in $b$ do affect the price dynamics when the steady state is unstable, but do not affect the stability of the steady state. To illustrate, we select $v=0.3, w=0.5$ and $\beta=1$. It follows from Table 3.1 that the corresponding Hopf bifurcation value ${ }^{11}$ is given by $g^{*}=1.4583$. Applying Proposition 3.2 to this bifurcation value, we obtain $d=0.1508-0.05169 b$, implying $d=0$ when $b=b^{*}=$ 2.917. Hence, the bifurcation is unstable for $b<b^{*}$ and stable for $b>b^{*}$, which can be verified numerically. Intuitively, when the fundamental price is unstable, the bifurcation is stable when the chartists are more concerned about the risk generated from the sample variance. Otherwise, the bifurcation becomes unstable and the price may become explosive.

In summary, when the chartists are trend followers, the stability of the fundamental steady state of the nonlinear deterministic system can be characterized by stability, bifurcation, and normal form analysis. It is found that the fundamental price is stable when traders are less active in extrapolation and switching between strategies. However, it becomes unstable when agents over-react to the price trend (for the chartists) and price dispersion (for the fundamentalists). Rational adaption of agents between strategies can lead market price to fluctuate around the fundamental price and to display complicated price dynamics (that are bifurcated from the Hopf bifurcation), including cycles, coexistence of cycles and strange attractors. In addition, when the fundamental price is unstable, the bifurcation becomes stable (unstable) when the trend followers are more (less) concerned about the risk generated from the sample variance. Also, because of the trend following strategy, prices tend to the positively correlated, as indicated by the phase plots in Figure 3.2.

\section{DYNAMICS OF FUNDAMENTALISTS AND CONTRARIANS}

In this section, we consider the situation in which the chartists are contrarians, that is, $g<0$ in model (2.19). Obviously the deterministic system has the same unique steady state, which corresponds to the fundamental price $p^{*}$. However, different from the previous case, the weight parameter leads to different dynamics.

Proposition 4.1. Assume $g<0$ for the deterministic system (2.19). There exist $w_{1}$ and $w_{2}$ satisfying $0<w_{1}<w_{2}<1 / 2$ such that:

(i) for $w>w_{2}$, the fundamental price $p^{*}$ of the deterministic system (2.19) is LAS if

$$
v>v_{2}(g):=-\frac{R\left(x_{0}+a\right)+a}{x_{0}}-\frac{2 a w}{x_{0}} g .
$$

\footnotetext{
${ }^{11}$ Since $C=0$, the bifurcation value $g^{*}$ is independent of $\beta$.
} 
In addition, if $v=v_{2}(g)$ and $\bar{d} \neq 0$ with

$$
\bar{d}:=\frac{1}{6} \frac{1}{3+2 \gamma_{1}-\gamma_{2}} \sum_{j, k, l=1}^{5} \frac{\partial^{3} F(0)}{\partial x_{j} \partial x_{k} \partial x_{l}}(-1)^{j+k+l+1},
$$

where

$$
\gamma_{1}=\frac{v x_{0}+a(1+g)}{R\left(x_{0}+a\right)}, \quad \gamma_{2}=-\frac{g a w}{R\left(x_{0}+a\right)},
$$

then a period two cycle bifurcates out of the steady state $X^{*}=0$. Furthermore, the bifurcation is stable when $\bar{d}<0$ and unstable when $\bar{d}>0$.

(ii) for $w<w_{1}$, the fundamental price $p^{*}$ of the deterministic system (2.19) is LAS if

$$
v>v_{3}(g):=\frac{c_{1}}{g}-c_{2}-c_{3} g .
$$

If $v=v_{3}(g)$, then there exists a pair of complex eigenvalues $\lambda=e^{ \pm i \theta}$ for the linearized system at the steady state. If, in addition, $d \neq 0$, where $d$ is defined by (3.2), then, in the absence of strong resonances (i.e., $e^{i k \theta} \neq 1$ for $k=1,2,3,4)$, a Hopf (Neimark-Sacker) bifurcation emanates out of the steady state $X^{*}=0$. Furthermore the bifurcation is supercritical when $d<0$ and subcritical when $d>0$.

(iii) for $w_{1} \leq w \leq w_{2}$, there exists a $g^{*}<0$ such that the fundamental price $p^{*}$ of the deterministic system (2.19) is LAS if

$$
v>v_{2}(g) \quad \text { for } \quad g \in\left(g^{*}, 0\right), \quad v>v_{3}(g) \quad \text { for } \quad g<g^{*} .
$$

In addition, for $g \in\left(g^{*}, 0\right)$ and $v=v_{2}(g)$, flip bifurcations occur when $\bar{d} \neq 0$, while for $g<g^{*}$ and $v=v_{3}(g)$ Hopf (Neimark-Sacker) bifurcations occur when $d \neq 0$. In addition, the bifurcation is stable (unstable) when $\bar{d}<0(\bar{d}>0)$ near the flip boundary and $d<0(d>0)$ near the Hopf bifurcation boundary.

Proof. See Appendix A.3.

Based on Proposition 4.1, the local stability region of the steady state and the corresponding bifurcation boundaries are different for the three different cases, as illustrated in $(g, v)$ parameter space in Figs. 4.1 and 4.2, respectively. The stability of the fundamental price is different from the case when the chartists are trend followers discussed in the previous section. The contrarian strategy can be viewed as a stable (unstable) force when the trend is weakly (strongly) extrapolated. Intuitively, the stability of the fundamental price depends on the balance of the activities from both types of traders. Note that both $v_{2}(g)$ and $v_{3}(g)$ decrease as $g$ increases. This implies that the stability region of the extrapolation rate of the contrarians (i.e. parameter $g$ ) is enlarged when the fundamentalists over-react (i.e. when $v$ increases). Intuitively, the fundamental price is stable as long as the forces from both types of traders are balanced. However, it is very interesting to notice that, different from the case when chartists are trend followers, the fundamental steady state price becomes unstable through either flip (for $w>w_{2}$ ), or Hopf (for $w<w_{1}$ ), or both types of bifurcation (for $w_{1}<w<w_{2}$ ), depending on the weight parameter $w$. 

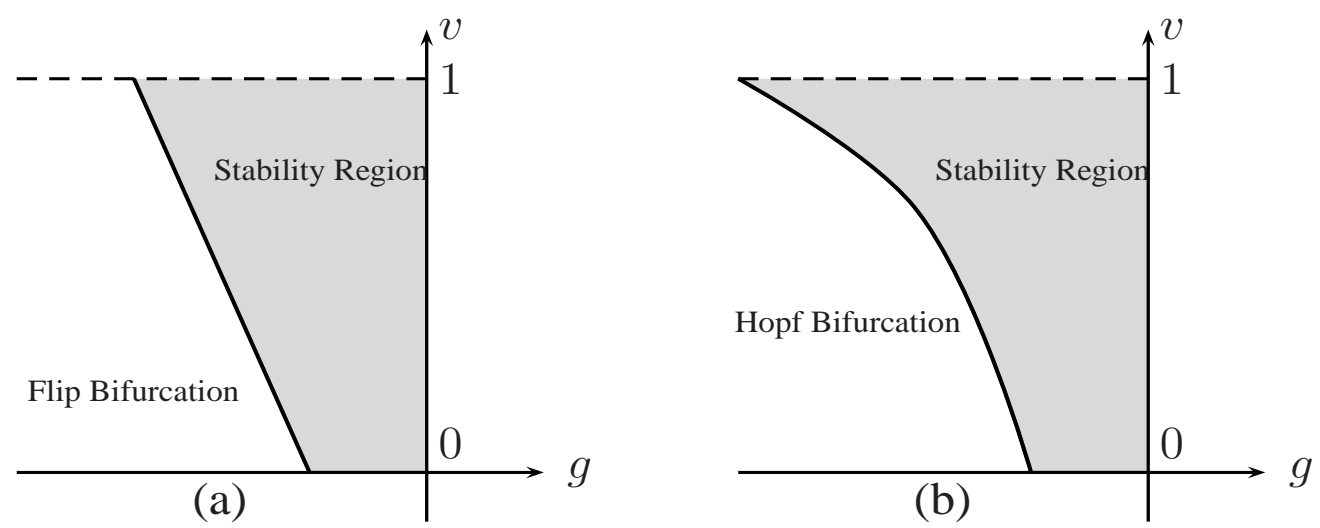

FIGURE 4.1. Local stability region of the steady state for $g<0$ and (a) the flip bifurcation boundary for $w \in\left[w_{2}, 1\right]$, (b) the Hopf bifurcation boundary for $w \in\left[0, w_{1}\right]$.

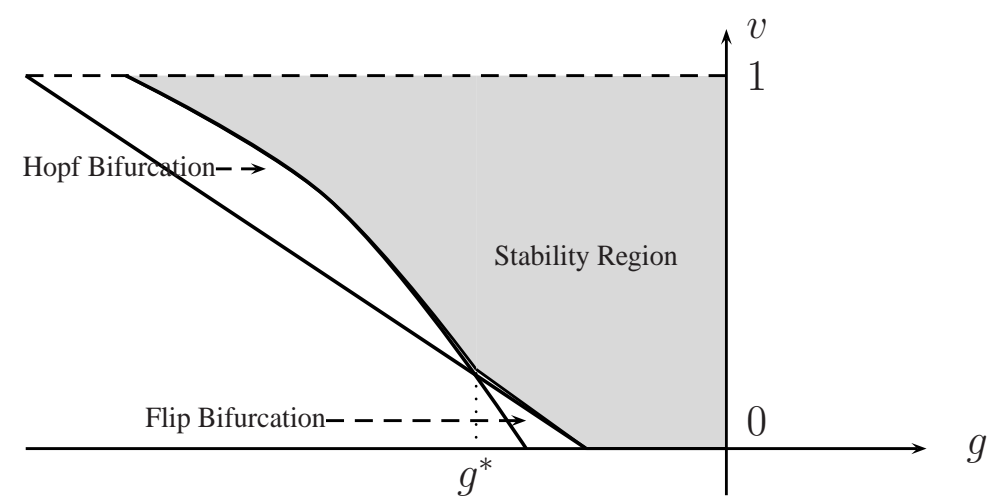

FIGURE 4.2. Local stability region of the steady state and the bifurcation boundaries for $g<0$ and $w \in\left(w_{1}, w_{2}\right)$.

When the contrarians put less weight (i.e. $w<w_{1}$ ) on the most recent price, the steady state fundamental price becomes unstable through a Hopf bifurcation. Intuitively, the extrapolation for the past price (with high weight) implies a sluggish reaction to the market price, leading the market price fluctuate periodically. For fixed $w=0.3$, Table 4.1 lists the Hopf bifurcation values $g_{h}^{*}$ and the corresponding values of $\rho$ for various $v$. One can see that, as $v$ increases, the stability region for the extrapolation rate $g_{h}^{*}$ is enlarged. Also, numerical calculations show that, as $w$ increases (up to $w=0.46$ ), the Hopf bifurcation is shifting to the left in Fig. 4.1(b). In other words, as the weight $w$ increases (up to a certain value), the stability region for the extrapolation rate is enlarged.

When the contrarians put more weight (i.e. $w>w_{2}$ ) on the most recent price, the steady state loses local stability through a flip bifurcation. Intuitively, a strongly negative extrapolation of the chartists to the most recent price (with high weight), 


\begin{tabular}{|c|c|c|c|c|c|c|c|c|c|c|c|}
\hline$v$ & 0 & 0.1 & 0.2 & 0.3 & 0.4 & 0.5 & 0.6 & 0.7 & 0.8 & 0.9 & 1 \\
\hline$g_{h}^{*}$ & -2.4597 & -2.4977 & -2.5361 & -2.5749 & -2.6142 & -2.6538 & -2.6938 & -2.7342 & -2.7749 & -2.8161 & -2.8576 \\
\hline$\rho$ & -1.5904 & -1.5727 & -1.5538 & -1.5384 & -1.5217 & -1.5054 & -1.4894 & -1.4738 & -1.4584 & -1.4434 & -1.4286 \\
\hline
\end{tabular}

TABLE 4.1. Hopf bifurcation values $g_{h}^{*}$ for various $v$ and $w=$ $0.3, a_{1}=a_{2}=0.8, \beta=0, C=0$.

together with the stabilizing force from the fundamentalists, causes the market price to flip back and forth around the fundamental price. For fixed $w=0.9$, Table 4.2 lists the flip bifurcation values $g_{f}^{*}$ for various $v$. As $v$ increases, the stability region for the extrapolation rate $g$ is enlarged. Numerical calculations also show that the flip bifurcation boundary in Fig. 4.1(a) is shifting to the left as $w$ increases, enlarging the stability region for the extrapolation rate.

\begin{tabular}{|c|c|c|c|c|c|c|c|c|c|c|c|}
\hline$v$ & 0 & 0.1 & 0.2 & 0.3 & 0.4 & 0.5 & 0.6 & 0.7 & 0.8 & 0.9 & 1 \\
\hline$g_{f}^{*}$ & -1.6669 & -1.7224 & -1.7780 & -1.8336 & -1.8891 & -1.9447 & -2.0002 & -2.0558 & -2.1113 & -2.1669 & -2.2224 \\
\hline
\end{tabular}

TABLE 4.2. Flip bifurcation values $g_{f}^{*}$ for various $v$ and $w=0.9, a_{1}=$ $a_{2}=0.8, \beta=0, C=0$.

When the weight parameter $w \in\left(w_{1}, w_{2}\right)$ with $0<w_{1}<w_{2}<1 / 2$, the fundamental steady state price loses its stability through both flip and Hopf bifurcations as indicated in Fig. 4.2. Table 4.3 lists the bifurcation values $g^{*}$ for various $w$ with fixed $v=0.5$. In this case, $w^{*} \in(0.4,0.5)$, which is determined by solving $v_{2}(g)=v_{3}(g)$ for $v=0.5, a_{1}=a_{2}=0.8, \beta=1, C=0$. The values $g^{*}$ correspond to Hopf bifurcation values for $w<w^{*}$ and flip bifurcation values for $w>w^{*}$. A similar result is also found in Chiarella and $\mathrm{He}(2003 a)$ when agents learn from a weighted average process. In other words, Hopf bifurcations occur when less weight is given to the most recent price, while flip bifurcations occur when more weight is given to the most recent price.

\begin{tabular}{|c||c|c|c|c|c|c|c|c|c|}
\hline$w$ & 0.1 & 0.2 & 0.3 & 0.4 & 0.5 & 0.6 & 0.7 & 0.8 & 0.9 \\
\hline$g^{*}$ & -2.0487 & -2.3127 & -2.6538 & -3.1110 & -3.5004 & -2.917 & -2.5003 & -2.1876 & -1.9447 \\
\hline
\end{tabular}

TABLE 4.3. Hopf bifurcation values $g^{*}$ for $w<w^{*}$ and flip bifurcation values $g^{*}$ for $w>w^{*}$ with $w^{*} \in(0.4,0.5)$ and $v=0.5, a_{1}=a_{2}=$ $0.8, \beta=1, C=0$.

Guided by the stability, bifurcation, and normal form analysis, we now examine the dynamics of the nonlinear system (2.19) by using numerical simulations.

We first examine the effect of extrapolation when the weighting parameter is low. To illustrate, we select $w=0.3, v=0.5, C=0, \beta=0, b=1$. In this case, the fundamental steady state is locally stable for $g>g_{h}^{*}=-2.6538$ and loses its stability through a Hopf bifurcation at $g=g_{h}^{*}$. Similarly, the nature of the Hopf bifurcation is determined by the values of $\rho$. In addition, using (3.2), one can verify that there exists a $b^{*} \in(0,1)$ such that $d<(>) 0$ for $b>(<) b^{*}$, implying that the bifurcations are stable for $b>b^{*}$ and unstable for $b<b^{*}$. Fig. 4.3 illustrates phase plots of the system for various values of $g$. It shows that, as the contrarians extrapolate weakly, the solutions converge to either the steady state (for $g>g^{*}$ ) or (quasi-)periodic cycles (for $g=-2.7,-4,-5<g^{*}$ ). The corresponding bifurcation is stable for $g$ near 

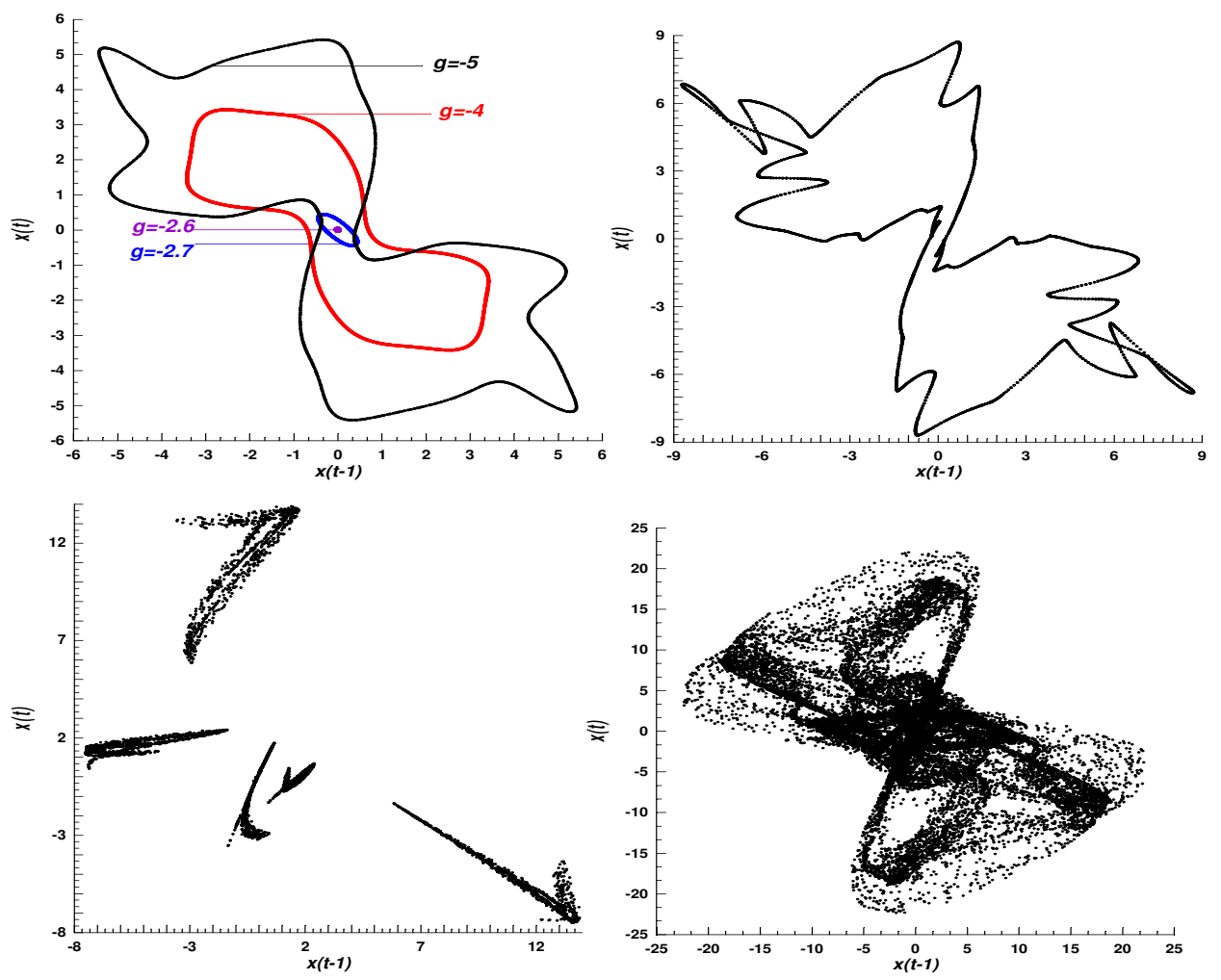

Figure 4.3. Phase plots of $\left(x_{t}, x_{t-1}\right)$ for $g=-2.6,-2.7,-4,-5$ (top left), $g=-6$ (top right), $g=-7$ (bottom left) and $g=-8$ (bottom right) with fixed $w=0.3, v=0.5, \beta=0, b=1, a_{1}=a_{2}=0.8, C=0$.

$g_{h}^{*}$. However, as the contrarians extrapolate more strongly, solutions converge to some strange (for $g=-6,-7$ ) and even chaotic (for $g=-8$ ) attractors. Similar to the case when the chartists are trend followers, coexistence of different cycles with different initial values for fixed $g=-2.65686$ is also found. The corresponding bifurcation diagram in parameter $g$ is plotted in Fig. 4.4.

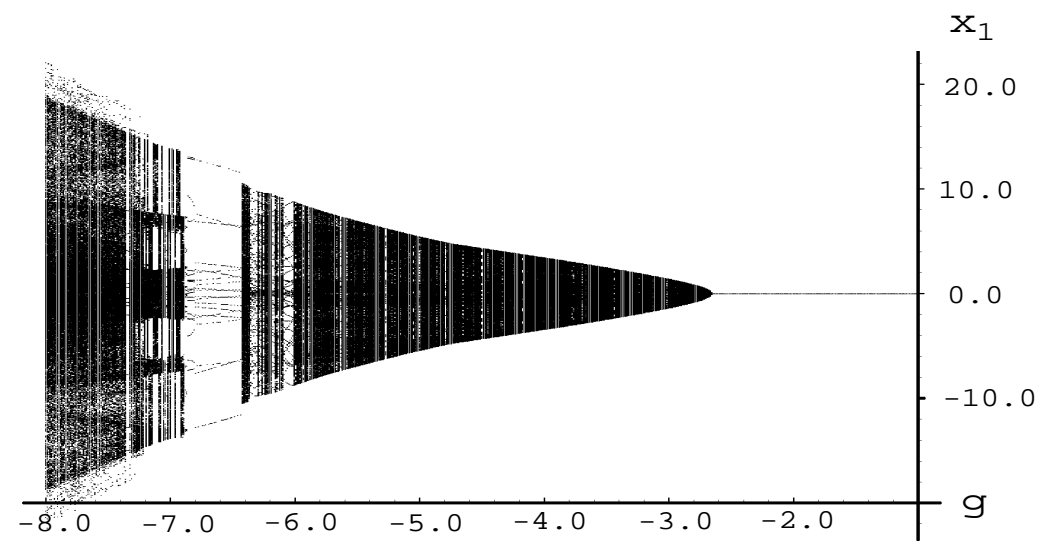

FIGURE 4.4. Bifurcation plot for the parameter $g$ with fixed $w=$ $0.3, v=0.5, \beta=0, b=1, a_{1}=a_{2}=0.8, C=0$. 
The effect of extrapolation when the weighting parameter is high can be different from the previous case. To illustrate, we choose $w=0.9$ and $v=0.5, C=0, \beta=$ $0, b=1$. Then the steady state is locally stable for $g>g_{f}^{*}=-1.9447$ and loses its stability through a flip bifurcation at $g=g_{f}^{*}$. Using (4.1), one can verify that $\bar{d}<0$ for all $b \geq 0$, implying that the bifurcated 2-period cycle is always stable for all $b \geq 0$ when $g=g_{f}^{*}$. Fig. 4.5 illustrates phase plots of the system for $g=$ $-3,-4,-5,-5.5,-6$. The corresponding bifurcation diagram for the parameter $g$ is given in Fig. 4.6. It shows that, as the contrarians increase their extrapolation activity, the dynamics lead to a period doubling bifurcation (for $g=-3$ ) first, and then two symmetric closed quasi-periodic cycles (for $g=-4$ ), and then to strange attractors (for $g=-5,-6$ ). Note that, for $g=-5.5$, solutions converge to two 14-periodic cycles. This shows a different route to complicated dynamics.
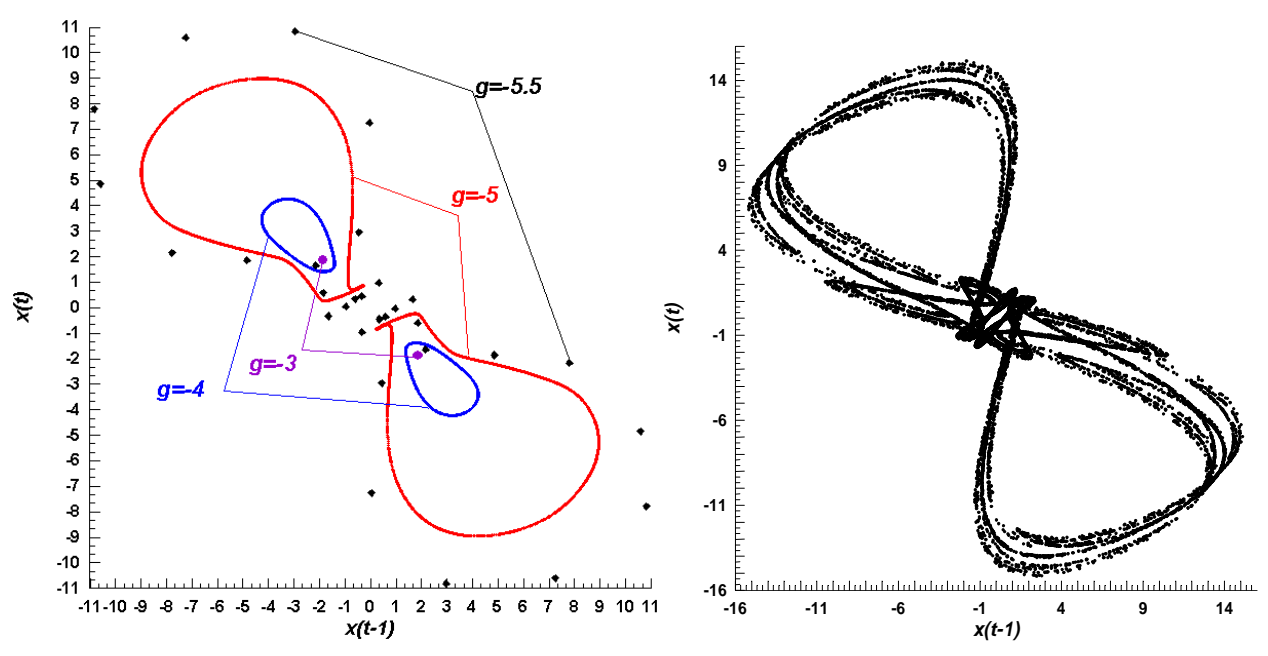

FIGURE 4.5. Phase plots of $\left(x_{t}, x_{t-1}\right)$ for $g=-3,-4,-5,-5.5$ (left) and $g=-6$ (right) with fixed $w=0.9, v=0.5, \beta=0, b=1, a_{1}=$ $a_{2}=0.8, C=0$.

The effect of switching intensity $\beta$ is also different. To illustrate, we choose $w=$ $0.48, v=0.5, C=0, b=1$. In this case the steady state is locally stable for $g>$ $g^{*}=-3.6057$ and a Hopf bifurcation occurs for $g=g^{*}$. For fixed $g=-6$, Fig. 4.7 illustrates phase plots of the system for $\beta=0$ (top left), $\beta=0.01$ (top right), $\beta=0.03$ (bottom left), and $\beta=0.1$ (bottom right). Different from the findings in Brock and Hommes (1998) and in the previous section, an increase in the switching intensity may stabilize the dynamics. This may due to the stabilizing nature of both fundamental and contrarian strategies.

In summary, for the model with contrarians, it is found that the fundamental price is stable when the stabilizing forces from both types of traders are balanced (low (high) extrapolations of the contrarians are balanced by under(over)-reactions of the fundamentalists). Otherwise, the fundamental price becomes unstable, leading to complicated dynamics through either flip, or Hopf, or both bifurcations, depending on the weight parameter. When the latest price is highly weighted, strong extrapolations from the contrarians cause the market price to flip back and forth around the fundamental price (through the flip bifurcation). When the latest price is weighted less, strong extrapolations from the contrarians generate lagged reaction of the market price to the 


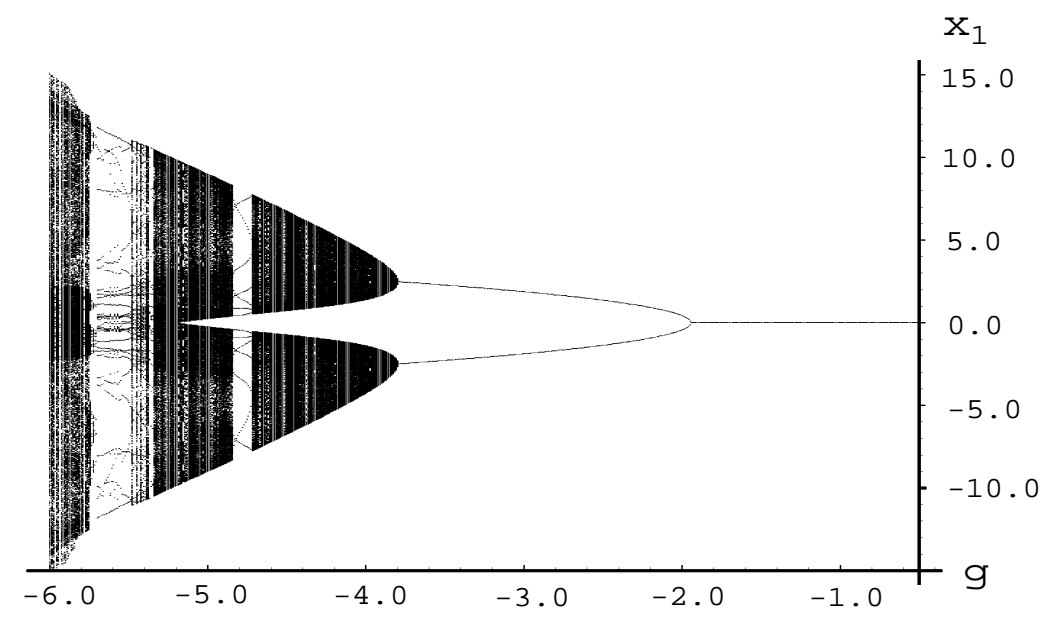

FIGURE 4.6. Bifurcation plot for the parameter $g$ with fixed $w=$ $0.9, v=0.5, \beta=0, b=1, a_{1}=a_{2}=0.8, C=0$.
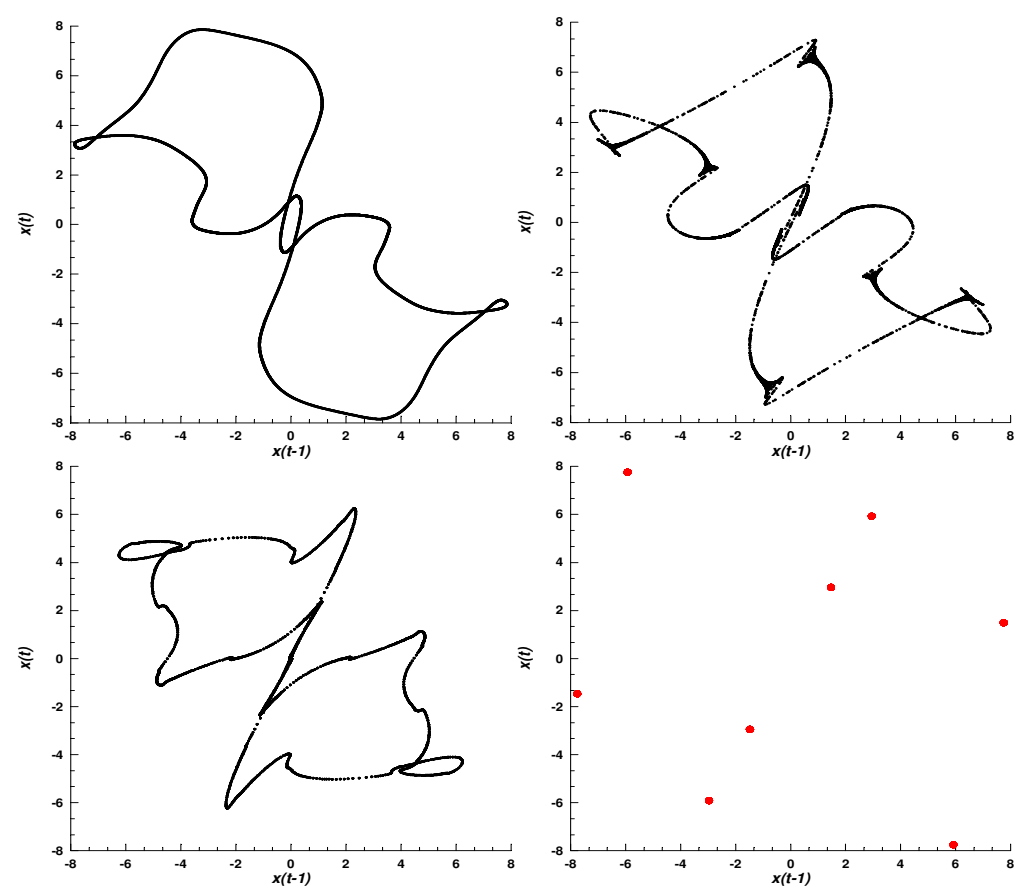

FIGURE 4.7. Phase plots of $\left(x_{t}, x_{t-1}\right)$ for $\beta=0$ (top left), $\beta=0.01$ (top right), $\beta=0.03$ (bottom left) and $\beta=0.1$ (bottom right) with fixed $w=0.48, v=0.5, g=-6, b=1$.

fundamental price, leading to periodic oscillation (through the Hopf bifurcation). The stability of the bifurcation depends on the parameter driven by the normal form analysis. Because of the stabilizing nature of both the fundamental and contrarian strategies, the price dynamics are less explosive, but more sensitive to the switching intensity between the two strategies. In addition, prices tend to be negatively correlated (as indicated by the phase plots) and high weight on the sample variance has a stabilizing effect. 


\section{CONCLUSIONS}

We present a simple asset pricing model with fundamentalists and chartists to study market price behaviour of the risky asset when chartists estimate both conditional mean and variance by using a weighted averaging process. Within our model, the fundamentalists represent a stabilizing force, the trend followers represent a destabilizing force, while the contrarians represent a stabilizing (destabilizing) force when they extrapolate weakly (strongly). Our analysis shows that the fundamental price is stable (unstable) when the different forces are balanced (unbalanced) and, when the fundamental price is unstable, there are different routes to complicated price dynamics. The weighting process has a different effect on the market price behaviour, depending on whether the chartists behave like trend followers or contrarians. When the chartists are trend followers, the fundamental price becomes unstable through Hopf bifurcation only for all values of the weighting parameter. However, when the chartists are contrarians, the fundamental price becomes unstable through a Hopf bifurcation only when the weighting parameter is low, a flip bifurcation only when the weighting parameter is high, and either Hopf or flip bifurcations when the weighting parameter is near 0.5. Using a normal form analysis, it is found that the time-varying second moment coefficient is clearly related to the stability of the bifurcations, rather than to the stability of the fundamental price. In principle, the bifurcation is stable (unstable) when the chartists are more (less) concerned on the risk generated from the time-varying second moment. To our knowledge, this is the first theoretical result on the role of a time-varying second moment on the stability of both the fundamental price and bifurcating orbits within the heterogeneous agent asset pricing framework.

The interplay between stochastic elements and deterministic dynamics is an interesting and important issue for model calibration. By assuming a random walk fundamental price process and introducing a noise trader to clear the market, a stochastic version of the deterministic model established in this paper is examined in Chiarella et al. (2005). The relationship between the statistical properties of the stochastic version and the stability and bifurcation of the underlying deterministic version, such as return autocorrelation patterns of the stochastic version and types of bifurcation of the underlying deterministic version, is examined. It is found that the model displays some of the stylised facts observed in high frequency financial data, such as fat tails, skewness and high kurtosis. The model also has the potential to generate volatility clustering and long memory features, which are the focus of much current research.

\section{Appendix A. Proof of Propositions}

A.1. Proof of Proposition 3.1. Let $X=X^{*}$ be the steady state of the corresponding deterministic system of (2.19). Then $X^{*}$ satisfies $X^{*}=G\left(X^{*}\right)$, which is equivalent to $x^{*}=F\left(x^{*}, x^{*}, x^{*}, x^{*}, x^{*}\right)$. Let

$$
m^{*}=\tanh \left[\frac{\beta}{2}(1-R)\left(\frac{v-R}{A_{1}}-\frac{1-R}{A_{2}}\right) x^{* 2}-\frac{\beta}{2} C\right] .
$$

Then $x^{*}$ satisfies

$$
x^{*}=\frac{v\left(1+m^{*}\right) x^{*}+a\left(1-m^{*}\right) x^{*}}{R\left(1+m^{*}\right)+R a\left(1-m^{*}\right)} .
$$

It follows that either $x^{*}=0$ or

$$
(R a-R+v-a) m^{*}=R A+R-v-a .
$$


If $R a-R+v-a=0$, then (A.2) cannot hold. If $R a-R+v-a \neq 0$, then

$$
m^{*}=\frac{R a+R-v-a}{R a-R+v-a}
$$

It is easy to see that $\left|m^{*}\right|>1$. Therefore $x^{*}=0$ is unique fixed point.

A.2. Proof of Proposition 3.2. Let $m^{0}=\tanh \left(-\frac{\beta}{2} C\right)$, and

$$
x_{0}=\frac{1+m^{0}}{1-m^{0}}=e^{-\beta C}>0
$$

We first examine the local stability and bifurcation. At the steady state $X=0$,

$$
\begin{aligned}
& \gamma_{1}=\left.\frac{\partial F}{\partial x_{1}}\right|_{X=0}=\frac{v x_{0}+a(1+g)}{R\left(x_{0}+a\right)}, \\
& \gamma_{2}=\left.\frac{\partial F}{\partial x_{2}}\right|_{X=0}=-\frac{g a w}{R\left(x_{0}+a\right)}, \\
& \gamma_{3}=\left.\frac{\partial F}{\partial x_{3}}\right|_{X=0}=-\frac{g a(1-w)}{R\left(x_{0}+a\right)}, \\
&\left.\frac{\partial F}{\partial x_{4}}\right|_{X=0}=0,\left.\quad \frac{\partial F}{\partial x_{5}}\right|_{X=0}=0 .
\end{aligned}
$$

Correspondingly, the Jacobin matrix of the system at the steady state is given by

$$
J:=\left(\begin{array}{ccccc}
\gamma_{1} & \gamma_{2} & \gamma_{3} & 0 & 0 \\
1 & 0 & 0 & 0 & 0 \\
0 & 1 & 0 & 0 & 0 \\
0 & 0 & 1 & 0 & 0 \\
0 & 0 & 0 & 1 & 0
\end{array}\right)
$$

Hence the characteristic equation satisfies

$$
\Gamma(\lambda):=\lambda^{2}\left(\lambda^{3}-\gamma_{1} \lambda^{2}-\gamma_{2} \lambda-\gamma_{3}\right)=0 .
$$

Define

$$
\pi(\lambda)=\lambda^{3}-\gamma_{1} \lambda^{2}-\gamma_{2} \lambda-\gamma_{3}
$$

Then two of the eigenvalues of $\Gamma(\lambda)$ are $\lambda_{4,5}=0$ (double), while the remaining eigenvalues satisfy $\pi(\lambda)=0$. Following from Jury's test (see, e.g. Elaydi (1996)), all the eigenvalues of $\pi(\lambda)$ satisfy $|\lambda|<1$ iff

(i) $\pi_{1}:=\pi(1)=1-\gamma_{1}-\gamma_{2}-\gamma_{3}>0$;

(ii) $\pi_{2}:=(-1)^{3} \pi(-1)=1+\gamma_{1}-\gamma_{2}+\gamma_{3}>0$;

(iii) $\pi_{3}:=1+\gamma_{2}+\gamma_{3}\left(\gamma_{1}-\gamma_{3}\right)>0$;

(iii) $\pi_{4}:=1-\gamma_{2}-\gamma_{3}\left(\gamma_{1}+\gamma_{3}\right)>0$.

Note that

$$
\begin{gathered}
\pi_{1}=\frac{(R-v) x_{0}+(R-1) a}{R\left(x_{0}+a\right)}>0, \\
\pi_{2}=\frac{(R+v) x_{0}+a(R+1+2 g w)}{R\left(x_{0}+a\right)}, \\
\pi_{3}=\frac{R^{2}\left(x_{0}+a\right)^{2}-g a w R\left(x_{0}+a\right)-\left(v x_{0}+a+g a\right) g a(1-w)-g^{2} a^{2}(1-w)^{2}}{R^{2}\left(x_{0}+a\right)^{2}},
\end{gathered}
$$

and

$$
\pi_{4}=\frac{R^{2}\left(x_{0}+a\right)^{2}+g a w R\left(x_{0}+a\right)+\left(v x_{0}+a+g a\right) g a(1-w)-g^{2} a^{2}(1-w)^{2}}{R^{2}\left(x_{0}+a\right)^{2}} .
$$

Hence $\pi_{1}>0$ is always satisfied. 
For $g>0, \pi_{2}>0$ and $\pi_{3}>0$ implies $\pi_{4}>0$. Hence, all four conditions are reduced to $\pi_{3}>0$. Therefore, the steady state is LAS if $\pi_{3}>0$.

For $w=1, \pi_{3}>0$ is reduced to the condition $g<g_{1} \equiv R\left(x_{o}+a\right) / a$ and hence the stability condition becomes $g<g_{1}$ for $w=1$. For $w \in[0,1)$, solving $v$ from the condition $\pi_{3}>0$ leads to

$$
v<v_{3}(g):=\frac{c_{1}}{g}-c_{2}-c_{3} g
$$

where $c_{1}, c_{2}$ and $c_{3}$ are defined as in Proposition 3.1. Hence the steady state is LAS if $v<v_{3}(g)$ for $w \in[0,1)$.

For $w \in[0,1)$, solving $g$ from $v=v_{3}(g)$ leads to

$$
g_{+}=\frac{1}{2 c_{3}}\left[-\left(c_{2}+v\right)+\sqrt{\left(c_{2}+v\right)^{2}+4 c_{1} c_{3}}\right] .
$$

One can verify that $g_{+} \rightarrow g_{1}$ as $w \rightarrow 1$.

It follows from $\pi_{1}>0$ and $\pi_{2}>0$ that both saddle-node and flip bifurcations cannot occur. Hence the steady state becomes unstable only through the Hopf bifurcation boundary $v=$ $v_{3}(g)$. Along the Hopf bifurcation boundary, the three eigenvalues must satisfy $\lambda_{1} \in(-1,1)$ and $\lambda_{2,3}=e^{ \pm \theta i}$. This leads to

$$
\rho+\lambda_{1}=\gamma_{1}, \quad 1+\rho \lambda_{1}=-\gamma_{2}, \quad \lambda_{1}=\gamma_{3},
$$

where $\rho=2 \cos (\theta)$. For $w \in[0,1)$, using $\pi_{3}=0$, one obtains that

$$
\rho=\gamma_{1}-\gamma_{3}=-\frac{1+\gamma_{1}}{\gamma_{3}}=\frac{R\left(x_{0}+a\right)-g a w}{g a(1-w)} .
$$

For $w=1$, the Hopf bifurcation boundary is given by $g=g_{1}, v \in[0,1]$. In this case, $\lambda_{1}=$ $\gamma_{3}=0, \gamma_{2}=-1$ and $\rho=\gamma_{1}=\left[v x_{o}+a\left(1+g_{1}\right)\right] /\left[R\left(x_{o}+a\right)\right]=1+\left(v x_{o}+a\right) /\left[R\left(x_{o}+a\right)\right]$. The nature of the Hopf bifurcation depends on $\theta$, and hence on the values of $\rho$. Note that, along the Hopf bifurcation boundary, $g$ is a function of $v$. Therefore types of Hopf bifurcation depend on the speed of the price adjustment of the fundamentalists towards the fundamental price, the extrapolation rate, and the weighting parameter $w$ of the trend followers.

We now conduct a normal form analysis to show the existence and stability of the Hopf bifurcation. Let the eigenvalues of $J$ be $\lambda_{1} \in(-1,1), \lambda_{2}=e^{i \theta}, \lambda_{3}=\bar{\lambda}_{2}$. Then

$$
\begin{aligned}
& \gamma_{3}=\lambda_{1}, \\
& \gamma_{1}=e^{i \theta}+e^{-i \theta}+\lambda_{1}, \\
& \gamma_{2}=1+\left(e^{i \theta}+e^{-i \theta}\right) \lambda_{1} .
\end{aligned}
$$

Let $q, p \in C^{5}$ be such that $J q=e^{i \theta} q, J^{T} p=e^{-i \theta} p$ and $\langle p, q\rangle=1$, where $\langle.,$.$\rangle is the$ usual inner product. Then we can take $q=\left(e^{4 i \theta}, e^{3 i \theta}, e^{2 i \theta}, e^{i \theta}, 1\right)^{T}$ and $p=\left(1, e^{-i \theta}-\right.$ $\left.\gamma_{1}, e^{i \theta} \gamma_{3}, 0,0\right)^{T} p_{1}$, where $p_{1}=\frac{e^{4 i \theta}}{2+e^{3 i \theta} \gamma_{3}-e^{i \theta} \gamma_{1}}$. A Neimark-Sarker bifurcation occurs if the following value is not equal to zero (see Kuznetsov (2004)):

$$
\begin{aligned}
d:= & \frac{1}{2} \operatorname{Re}\left\{e ^ { - i \theta } \left[\langle p, C(q, q, \bar{q})\rangle+2\left\langle p, B\left(q,(E-A)^{-1} B(q, \bar{q})\right\rangle\right.\right.\right. \\
& \left.+\left\langle p, B\left(\bar{q},\left(e^{2 i \theta} E-A\right)^{-1} B(q, q)\right\rangle\right]\right\},
\end{aligned}
$$

where $C(q, q, q), B(q, q) \in R^{5}$ and

$$
\begin{gathered}
C_{i}(q, q, q)=\sum_{j, k, l=1}^{5} \frac{\partial^{3} G_{i}(0)}{\partial x_{j} \partial x_{k} \partial x_{l}} q_{j} q_{k} q_{l}, \\
B_{i}(q, q)=\sum_{j, k=1}^{5} \frac{\partial^{2} G_{i}(0)}{\partial x_{j} \partial x_{k}} q_{j} q_{k},
\end{gathered}
$$


$E$ is the identity matrix. Note that $\frac{\partial^{2} G_{i}(0)}{\partial x_{j} \partial x_{k}}=0, i, j, k=1, \ldots 5$. Hence

$$
\begin{aligned}
d & =\frac{1}{2} \operatorname{Re}\left\{e^{-i \theta}[\langle p, C(q, q, \bar{q})\rangle]\right\} \\
& =\frac{1}{2} \frac{1}{e^{-3 i \theta}-e^{-i \theta}+\gamma_{3}\left(1-e^{-2 i \theta}\right)} \sum_{j, k, l=1}^{5} \frac{\partial^{3} F(0)}{\partial x_{j} \partial x_{k} \partial x_{l}} e^{(j+k-l-5) i \theta} .
\end{aligned}
$$

A.3. Proof of Proposition 4.1. Following from the first part of the proof of Proposition 3.1 in Appendix A.2, all the three eigenvalues of $\pi(\lambda)=0$ satisfy $|\lambda|<1$ iff $\pi_{i}>0$ for $i=1,2,3,4$. $\pi_{1}>0$ is always satisfied. Hence the stability boundaries of the local stability region of the steady state depend on the relative positions of $\pi_{2}=\pi_{2}=\pi_{4}=0$ on the $(g, v)$ parameter plane. Solving for $v$ in terms of $g$ from $\pi_{2}=\pi_{3}=\pi_{4}=0$ leads to the following equations, respectively,

$$
\begin{aligned}
& v_{2}(g)=-\frac{R\left(x_{0}+a\right)+a}{x_{0}}-\frac{2 a w}{x_{0}} g, \\
& v_{3}(g)=c_{1} \frac{1}{g}-c_{2}-c_{3} g, \\
& v_{4}(g)=-c_{1} \frac{1}{g}-c_{2}-\frac{a w}{x_{0}} g .
\end{aligned}
$$

For $w=1, \pi_{2}>0, \pi_{3}>0$ and $\pi_{4}>0$ are equivalent to

$$
g>-\frac{1}{2 a}\left[R\left(x_{o}+a\right)+v x_{0}+a\right], \quad g<\frac{R\left(x_{o}+a\right)}{a}, \quad g>-\frac{R\left(x_{o}+a\right)}{a},
$$

respectively. Given $v \in[0,1]$ and $R>1$, these three conditions are reduced to $g>-\frac{1}{2 a}\left[R\left(x_{o}+\right.\right.$ $\left.a)+v x_{0}+a\right]$, which is equivalent to

$$
v>-\frac{R\left(x_{o}+a\right)+a+2 a g}{x_{o}} .
$$

In addition, along the boundary, one of the eigenvalues is -1 , and hence a flip bifurcation occurs.

Now let $w \in[0,1)$. Note that $v=v_{2}(g)$ is a straight line with slope $-\frac{2 a w}{x_{0}}$, which is decreasing from 0 to $-\frac{2 a}{x_{0}}$ as $w$ varies from 0 to $1 ; v=v_{3}(g)$ is concave up and decreasing from $+\infty$ to $-\infty$ for $g \in(-\infty, 0)$, and it moves to the left as $w$ varies from 0 to $1 ; v=v_{4}(g)$ is concave down and $\lim _{g \rightarrow-\infty} v_{4}(g)=\lim _{g \rightarrow 0^{-}} v_{4}(g)=+\infty$.

The relative position of the two curves $v=v_{3}(g)$ and $v=v_{4}(g)$ is determined by

$$
v_{43}=v_{4}(g)-v_{3}(g)=-2 c_{1} \frac{1}{g}+\left(c_{3}-\frac{a w}{x_{0}}\right) g .
$$

Hence $v_{43}=0$ implies that $g=g_{43}=-\frac{\left(x_{0}+a\right) R}{a(1-w)}$. Note that

$$
v_{4}\left(g_{43}\right)=v_{3}\left(g_{43}\right)=\frac{\left(x_{0}+a\right) R-a}{x_{0}}>1 \text {. }
$$

Therefore two curves $\pi_{3}=0$ and $\pi_{4}=0$ intersect at one point, which is always above the line $v=1$. It is easy to see that $\frac{\partial v_{4}}{\partial g}\left(g_{43}\right)=a(1-2 w) / x_{0}<0,=0,>0$ if $w>\frac{1}{2},=\frac{1}{2},<\frac{1}{2}$ respectively. The relative positive positions of those curves are plotted in Fig. A.1. Obviously, for $w \leq 1 / 2$, the stability conditions are reduced to $\pi_{2}>0$ and $\pi_{3}>0$. It can be shown ${ }^{12}$ that this is also true for $w>1 / 2$. Therefore, the boundaries of the stability of the steady state are determined by $\pi_{2}=\pi_{3}=0$.

\footnotetext{
${ }^{12}$ In fact, $\pi_{2}=\pi_{4}=0$ intersect at $g=g_{24}=-R\left(x_{0}+a\right) /(a w)$. Note that $v_{4}^{\prime}\left(g_{24}\right)=a w(2 w-$ $1) /\left(x_{0}(1-w)\right)$, which is positive for $w>1 / 2$ and negative for $w<1 / 2$. Hence, for $w>1 / 2$, if $g_{24}<g<0$, the curve $\pi_{4}=0$ is above the curve $\pi_{2}=0$. Therefore, $\pi_{4}>0$ when $v \leq 1$ and $g>g_{24}$. The stability boundaries are then determined by $v=0, v=1$ and $\pi_{2}=0$.
} 


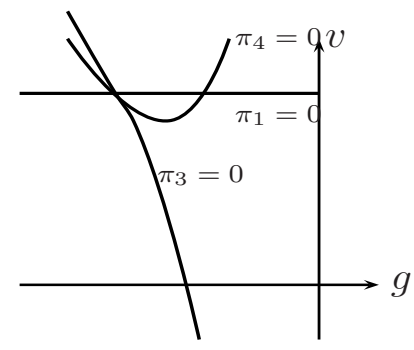

$w>\frac{1}{2}$

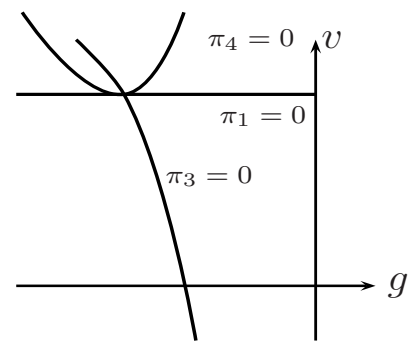

$w=\frac{1}{2}$

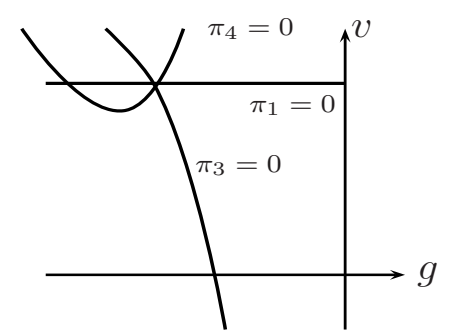

$w<\frac{1}{2}$

FIgURE A.1. Relative position of $\pi_{i}=0$ for $i=1,3,4$.

The relative position of the curves $v=v_{2}(g)$ and $v=v_{3}(g)$ is determined by

$$
v_{32}=v_{3}(g)-v_{2}(g)=\frac{(x+a)^{2} R^{2}}{a x_{0}(1-w)} \frac{1}{g}+\frac{\left(R x_{0}+a\right)(1-2 w)}{x_{0}(1-w)}+\frac{a(3 w-2)}{x_{0}} g .
$$

It is easy to see that, for $g<0, v_{32}<0$ for $w \geq \frac{2}{3}$, and $v=v_{32}(g)$ is decreasing from $+\infty$ to $-\infty$ for $g \in(-\infty, 0)$ if $w \in\left[0, \frac{2}{3}\right)$. Therefore, when $w \geq \frac{2}{3}$, the line $v=v_{2}(g)$ is above the curve $v=v_{3}(g)$. Hence the stability conditions are reduced to $\pi_{2}>0$ and the stability region is bounded by a flip bifurcation boundary only. When $w<\frac{2}{3}$, there is a unique intersection for these two curves, and the relative position is shown as Figure A.2. Consequently, there exist $w_{1}$ and $w_{2}$ satisfying $0<w_{1}<w_{2}<2 / 3$ such that the intersection of these two curves $v=v_{2}(g)$ and $v=v_{3}(g)$ is located in the banded region $0 \leq v \leq 1$ if $w_{1} \leq w \leq w_{2}$.
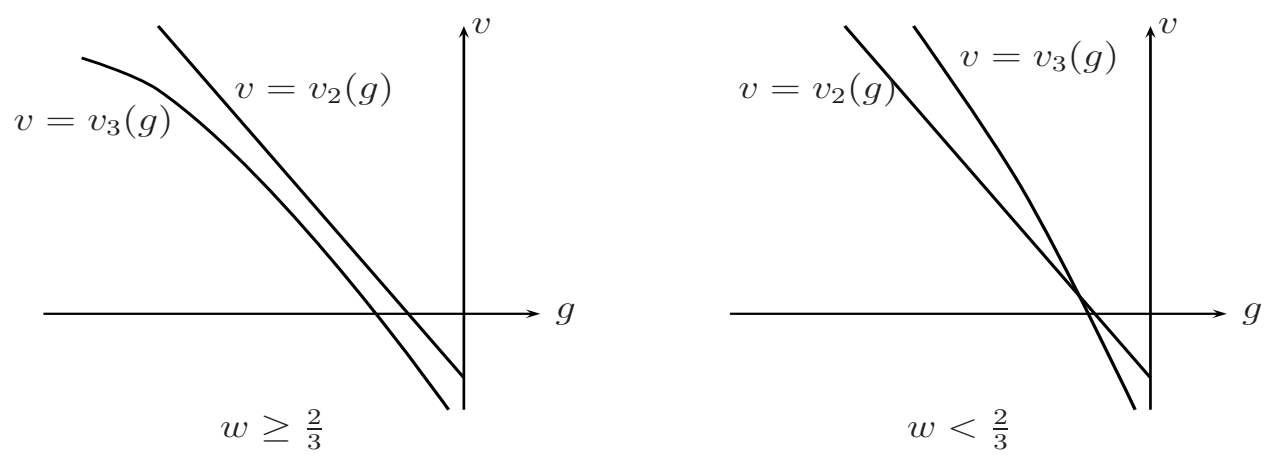

Figure A.2. The relative position of $v=v_{2}(g)$ and $v=v_{3}(g)$ for both $w \geq 2 / 3$ and $w<2 / 3$.

Note that the curves $v=v_{1}(g)$ and $v=v_{2}(g)$ intersect when $g=g_{12}=-\left(2 R x_{0}+R a+\right.$ a)/(2aw). Obviously, $g_{12}$ increases and $g_{43}{ }^{1}$ decreases for $w \in(0,1)$. Also, $g_{12}<g_{43}$ for $w=0$ and $g_{12}>g_{43}$ for $w=1 / 2$. Hence $w_{2}<1 / 2$.

We now conduct the normal form analysis. The matrix $J$ has an eigenvalue of -1 if and only if $\gamma_{3}=-1-\gamma_{1}+\gamma_{2}$. Now we assume that this condition holds. Then a flip bifurcation may occur, depending on the value of coefficient of the third order term in the normal form. We now give the computation for the flip bifurcation. 
Let $q=(1,-1,1,-1,1)^{T}$ and $p=\left(1,-1-\gamma_{1}, 1+\gamma_{1}-\gamma_{2}, 0,0\right)^{T} /\left(3+2 \gamma_{1}-\gamma_{2}\right)$. Then $A q=-q, A^{T} p=-p$ and $\langle p, q\rangle=1$. The type of the flip bifurcation depends on the value

$$
\bar{d}:=\frac{1}{6}\langle p, C(q, q, q)\rangle-\frac{1}{2}\left\langle p, B\left(q,(A-E)^{-1} B(q, q)\right)\right\rangle,
$$

where $C(q, q, q), B(q, q) \in R^{5}$ are defined in the previous proof. Note that $\frac{\partial^{2} G_{i}(0)}{\partial x_{j} \partial x_{k}}=0, i, j, k=$ $1, \ldots 5$. So $\bar{d}=\frac{1}{6}\langle p, C(q, q, q)\rangle$. Hence

$$
\bar{d}=\frac{1}{6} \frac{1}{3+2 \gamma_{1}-\gamma_{2}} \sum_{j, k, l=1}^{5} \frac{\partial^{3} F(0)}{\partial x_{j} \partial x_{k} \partial x_{l}}(-1)^{j+k+l+1} .
$$

The normal form calculation for the Hopf bifurcation is given in the proof of Proposition 3.2 in Appendix A.2.

\section{REFERENCES}

Anderson, S. and A. de Palma and J. Thisse (1993), Discrete Choice Theory of Product Differentiation, MIT Press, Cambridge, MA.

Arthur, W., Holland, J., LeBaron, B., Palmer, R. and Tayler, P. (1997), 'Asset pricing under endogeneous expectations in an artifical stock market', Economic Notes 26(2), 297-330.

Bollerslev, T., Engle, R. and Nelson, D. (1994), ARCH models, Handbook of Econometrics, Elsevier, Amsterdam, pp. 2959-3038.

Brock, W. and Hommes, C. (1997a), Models of Complexity in Economics and Finance, Wiley, New York, chapter 1, pp. 3-44. in Systems Dynamics in Economic and Finance Models, Eds. Heij, C., Schumacher, J.M., Hanzon, B., Praagman, C.

Brock, W. and Hommes, C. (1997b), 'A rational route to randomness', Econometrica 65, 1059-1095.

Brock, W. and Hommes, C. (1998), 'Heterogeneous beliefs and routes to chaos in a simple asset pricing model', Journal of Economic Dynamics and Control 22, 1235-1274.

Brock, W. and LeBaron, B. (1996), 'A structural model for stock return volatility and trading volume', Review of Economics and Statistics 78, 94-110.

Bullard, J. and Duffy, J. (1999), 'Using Genetic Algorithms to Model the Evolution of Heterogeneous Beliefs', Computational Economics 13, 41-60.

Chen, S.-H. and Yeh, C.-H. (1997), 'Toward a computable approach to the efficient market hypothesis: an application of genetic programming', Journal of Economic Dynamics and Control 21, 10431063.

Chen, S.-H. and Yeh, C.-H. (2002), 'On the emergent properties of artificial stock markets: the efficient market hypothesis and the rational expectations hypothesis', Journal of Economic Behavior and Organization 49, 217-239.

Chiarella, C. (1992), 'The dynamics of speculative behaviour', Annals of Operations Research 37, 101123.

Chiarella, C., Dieci, R. and Gardini, L. (2002), 'Speculative behaviour and complex asset price dynamics', Journal of Economic Behavior and Organization 49, 173-197.

Chiarella, C. and He, X. (2001), 'Asset pricing and wealth dynamics under heterogeneous expectations', Quantitative Finance 1, 509-526.

Chiarella, C. and He, X. (2002), 'Heterogeneous beliefs, risk and learning in a simple asset pricing model', Computational Economics 19, 95-132.

Chiarella, C. and He, X. (2003a), Economic Complexity: Non-linear Dynamics, Multi-Agents Economies, and Learning, Vol. 14 of ISETE (International Symposium in Economic Theory and Econometrics) Series, Elsevier, chapter Dynamics of Beliefs and Learning under $a_{L}$-Processes The Homogeneous Case, pp. 363-390.

Chiarella, C. and He, X. (2003b), 'Heterogeneous beliefs, risk and learning in a simple asset pricing model with a market maker', Macroeconomic Dynamics 7, 503-536.

Chiarella, C., He, X. and Wang, D. (2005), Economics and Heterogeneous Interacting Agents, Eds. A. Namatame, T. Kaizoji and Y. Aruka, Lecture Notes in Economics and Mathematical Systems, 
Springer, chapter Statistical Properties of a heterogeneous asset price model with time-varying second moment. in press.

Dacorogna, M., Muller, U., Jost, C., Pictet, O., Olsen, R. and Ward, J. (1995), 'Heterogeneous real-time trading strategies in the foreign exchange market', European Journal of Finance 1, 383-403.

Day, R. and Huang, W. (1990), 'Bulls, bears and market sheep', Journal of Economic Behavior and Organization 14, 299-329.

Elaydi, S. (1996), An Introduction to Difference Equations, Springer, New York.

Farmer, J. and Joshi, S. (2002), 'The price dynamics of common trading strategies', Journal of Economic Behavior and Organization 49, 149-171.

Gaunersdorfer, A. (2000), 'Endogenous fluctuations in a simple asset pricing model with heterogeneous agents', Journal of Economic Dynamics and Control 24, 799-831.

Gaunersdorfer, A., Hommes, C. and Wagener, F. (2003), Bifurcation routes to volatility clustering under evolutionary learning, Technical Report 03-03, CeNDEF, University of Amsterdam. Working Paper.

Grossman, S. (1989), The Informational Role of Prices, MIT Press, Cambridge, MA.

Hommes, C. (2001), 'Financial markets as nonlinear adaptive evolutionary systems', Quantitative Finance 1, 149-167.

Hommes, C. (2002), 'Modeling the stylized facts in finance through simple nonlinear adaptive systems', Proceedings of National Academy of Science of the United States of America 99, 7221-7228.

Kirman, A. (1992), 'Whom or what does the representative agent represent?', Journal of Economic Perspectives 6, 117-136.

Kuznetsov, Y. (2004), Elements of applied bifurcation theory, Vol. 112 of Applied mathematical sciences, 3rd edn, SV, New York.

LeBaron, B., Arthur, W. and Palmer, R. (1999), 'Time series properties of an artifical stock market', Journal of Economic Dynamics and Control 23, 1487-1516.

Lux, T. (1995), 'Herd behaviour, bubbles and crashes', Economic Journal 105, 881-896.

Lux, T. (1997), 'Time variation of second moments from a noise trader/infection model', Journal of Economic Dynamics and Control 22, 1-38.

Lux, T. (1998), 'The socio-economic dynamics of speculative markets: Interacting agents, chaos, and the fat tails of return distributions', Journal of Economic Behavior and Organization 33, 143-165.

Lux, T. and Marchesi, M. (1999), 'Scaling and criticality in a stochastic multi-agent model of a financial market', Nature 397(11), 498-500.

Manski, C. and McFadden, D. (1981), Structural Analysis of Discrete Data with Econometric Applications, MIT Press.

Nelson, D. (1992), 'Filtering and forecasting with missepecified ARCH models I: getting the right variance with the wrong model', Journal of Economics 52, 61-90. 